\title{
Influencia de la interacción suelo estructura (ISE) de cimentaciones superficialesen suelos no cohesivos en el comportamiento estructural de una edificación de 8 pisos y un subsuelo
}

Influence of The Soil Structure Iteraction (Ise) on Surface Foundations and Cohesive Soils In The Structural Behavior of an Eight-Story Basement Building

\author{
Morales L. ${ }^{1}$; Espinosa A. ${ }^{2}$ \\ 1 Universidad Central del Ecuador, Facultad de Ingeniería, Ciencias Físicas y Matemática. Quito, Ecuador \\ e-mail: lwmorales@uce.edu.ec \\ 2 Universidad Central del Ecuador, Facultad de Ingeniería en Geología, Minas, Petróleos y Ambiental. Quito, \\ Ecuador \\ e-mail: alejandrostead@hotmail.com
}

Información del artículo

Recibido: febrero 2020

Aceptado: marzo 2020

\begin{abstract}
RESUMEN
Se comparan periodos de vibración y derivas de pisos obtenidos de modelos tradicionales con base rígida (empotramiento perfecto), y otros, donde el suelo se representa con funciones de impedancia y, directamente mediante elementos finitos no lineales (método directo). Se usa como proyecto base una edificación de ocho niveles y un subsuelo, que interactúa con un suelo no cohesivo blando. Para las cargas sísmicas se consideraron: el espectro de la Norma Ecuatoriana de la Construcción y el de sitio, que corresponde de acuerdo con la ubicación del proyecto, al generado por el segmento de la falla de Quito denominado Puengasí. Adicionalmente, se usan historias en el tiempo escaladas de acuerdo con el espectro de sitio. El comportamiento no lineal de la estructura se logra por medio de rótulas plásticas. Se observa que el modelo que incluye interacción mediante funciones de impedancia varía hasta un $45 \%$ respecto al de base rígida, sin embargo, da resultados conservadores respecto al método directo.
\end{abstract}

Palabras clave: interacción suelo estructura, espectro de respuesta, periodo de vibración de la estructura y derivas de piso.

\begin{abstract}
Vibration periods and floor drifts are obtained from traditional models with a rigid base (perfect embedment), and others, where the floor is represented with impedance functions and, directly by finite non-linear elements (direct method). An eight-level building and a subsoil is used as the base project, which interacts with a soft non-cohesive soil. For the seismic loads were considered: the spectrum of the Ecuadorian Standard of Construction and the site, which corresponds according to the location of the project, to the one generated by the segment of the Quito fault called Puengasí. Additionally, time-scaled stories are used according to the site spectrum. The non-linear behavior of the structure is achieved by means of plastic ball joints. It is observed that the model that includes interaction through impedance functions varies up to $45 \%$ with respect to the rigid one, however, it gives conservative results with respect to the direct method.
\end{abstract}

Keywords: soil structure soil, response spectrum, period of structure vibration and story drifts. 


\section{Introducción}

Para el diseño estructural de edificaciones se emplean apoyos de base rígida, el cual presenta una rigidez infinita provocando una reducción de los movimientos de entrada de un terremoto, que se traduce en periodos de vibraciones de la estructura menores a los reales. La interacción suelo estructura (ISE) por otro lado considera un apoyo flexible, que genera movimientos de entrada más reales.

Modelar a la cimentación como empotramiento perfecto es una simplificación de la ISE y considera que el suelo es rígido. Un suelo es rígido dependiendo del valor Vs30 (velocidades de ondas de corte a 30m de profundidad) y la clasificación propuesta por [1] es empleada por muchos códigos de sismo resistencia.

Tabla 1. Clasificación de perfiles de suelos

\begin{tabular}{clll}
\cline { 1 - 2 } Tipo & & Descripción & Vs30 m/s \\
\cline { 1 - 2 } A & Roca rígida & $>1500$ \\
\hline B & Roca de rigidez media & 760 a 1500 \\
\hline C & Suelos muy densos o roca & 360 a 760 \\
\hline D & Suelos rígidos & 180 a 360 \\
\hline E & Arcillas blandas & $<180$ \\
\hline F & Suelos especiales & \\
\hline
\end{tabular}

En suelos blandos y especiales conlleva un error el modelar a la cimentación como empotramiento perfecto, todo esto debido a que los valores del módulo cortante máximo y secante (Gmax y Gsec) son pequeños. Una manera fácil de estimarlos es mediante la siguiente ecuación:

$$
\mathrm{G}_{\max }=\frac{\rho V_{S}^{2}}{\mathrm{~g}}
$$

$$
\mathrm{Gsec}=\frac{\mathrm{G}}{\mathrm{Gmax}} \mathrm{Gmax}
$$

Donde:

Gmax : $\quad$ Módulo de corte máximo

$\rho \quad: \quad$ Densidades del suelo

Vs : Velocidad de ondas de corte
G/Gmax puede estimarse de igual manera en función de la clasificación del perfil de suelos [2].

Tabla 2. Tabla de Módulo cortante efectivo G/Gmax

\begin{tabular}{ccccc}
\hline \multicolumn{5}{c}{ Picodeaceleraciones efectivas PGA (a) } \\
\hline Tipo & PGA $=0$ & PGA $=0.1$ & PGA $=0.4$ & PGA $=0.8$ \\
\hline A & 1 & 1 & 1 & 1 \\
B & 1 & 1 & 0.95 & 0.9 \\
C & 1 & 0.95 & 0.75 & 0.6 \\
D & 1 & 0.9 & 0.5 & 0.1 \\
E & 1 & 0.6 & 0.05 & (b) \\
F & (b) & (b) & (b) & (b) \\
\hline
\end{tabular}

(a) Use interpolación lineal para valores intermedios de PGA.

(b) Se realizarán investigaciones geotécnicas específicas del sitio y análisis dinámicos.

Gmax y Gsec se obtienen directamente de ensayos cíclicos de suelos como triaxiales, ensayos de corte directo, ensayos de corte torsional y columna resonante.

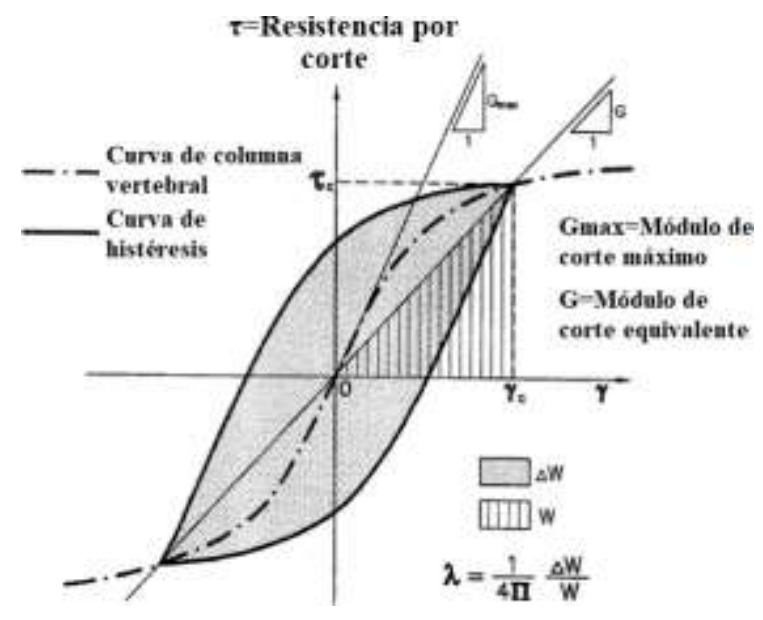

Figura 1. Curva histéresis del suelo

\subsection{Método de la subestructura - Modelo B}

El método de la subestructura se basa en representar a la cimentación como un apoyo rígido o por medio de resortes.

El análisis con resortes emplea las funciones de impedancia a partir Pais-Kausel [2]: 


$$
\begin{array}{lllll}
K_{z}=\left(\frac{G . B}{2-v}\right)\left(3.1\left(\frac{L}{B}\right)^{0.75}+1.6\right) & C & : & \text { Amortiguamiento } \\
& \text { (3) } & \beta & : & \text { Amortiguamiento por radiación } \\
K_{y}=\left(\frac{G . B}{2-v}\right)\left(6.8\left(\frac{L}{B}\right)^{0.65}+0.8\left(\frac{L}{B}\right)+1.6\right) & \text { (4) } & K & : & \text { Rigidez }
\end{array}
$$$$
K_{x}=\left(\frac{G . B}{2-v}\right)\left(6.8\left(\frac{L}{B}\right)^{0.65}+2.4\right)
$$$$
K_{z z}=\left(G . B^{3}\right)\left(4.25\left(\frac{L}{B}\right)^{2.45}+4.06\right)
$$$$
K_{y y}=\left(\frac{G \cdot B^{3}}{1-v}\right)\left(3.73\left(\frac{L}{B}\right)^{2.4}+0.27\right)
$$$$
K_{x x}=\left(\frac{G \cdot B^{3}}{1-v}\right)\left(3.2\left(\frac{L}{B}\right)+0.8\right)
$$$$
C_{z}=\frac{2 K_{z}\left(0.05+\beta_{z}\right)}{w}
$$$$
C_{z}=\frac{2 K_{y}\left(0.05+\beta_{y}\right)}{w}
$$$$
C_{X}=\frac{2 K_{X}\left(0.05+\beta_{X}\right)}{w}
$$$$
C_{Z Z}=\frac{2 K_{Z Z}\left(0.05+\beta_{Z Z}\right)}{w}
$$$$
C_{Y Y}=\frac{2 K_{Y Y}\left(0.05+\beta_{Y Y}\right)}{w}
$$$$
C_{X X}=\frac{2 K_{X X}\left(0.05+\beta_{X X}\right)}{w}
$$

\subsection{Método directo de ISE-Modelo C}

Para emplear este método es necesario escoger un modelo de elasto-plasticidad adecuado del suelo. El modelo Hardening Soil with small-strain stiffness HSSMALL se ajusta al tipo de suelo encontrado en las investigaciones de campo y es necesarios definir los siguientes parámetros:

m : Dependencia de la rigidez al estado de esfuerzos.

$E_{50}^{\text {ref }}:$ Módulo elasticidad secante en ensayos triaxiales.

$E_{e o d}^{r e f}:$ Módulo de elasticidad tangente para carga primaria del ensayo edométrico.

$\begin{array}{ll}E_{u r}^{\text {ref }}: & \text { Rigidez de carga y descarga en ensa- } \\ & \text { yos triaxiales cíclicos }\end{array}$

$v_{u r}$ : Relación de Poisson para carga y descarga.

$G_{0}^{r e f}:$ Módulo de corte máximo en deformaciones muy pequeñas

$\gamma_{0.7}:$ Tensión de corte de umbral en la que $\mathrm{G}=0.722 \mathrm{Go}$ (15).

Según [3, p. 52] este parámetro se define grafican-

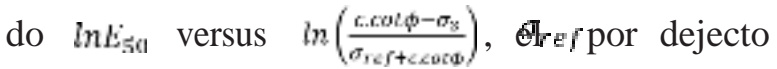
tiene un valor de $100 \mathrm{KPa}(1.02 \mathrm{~kg} / \mathrm{cm} 2)$.

Donde:

G : $\quad$ Gsec

B : $\quad$ Base de lacimentación

L : : Largo de la cimentación 


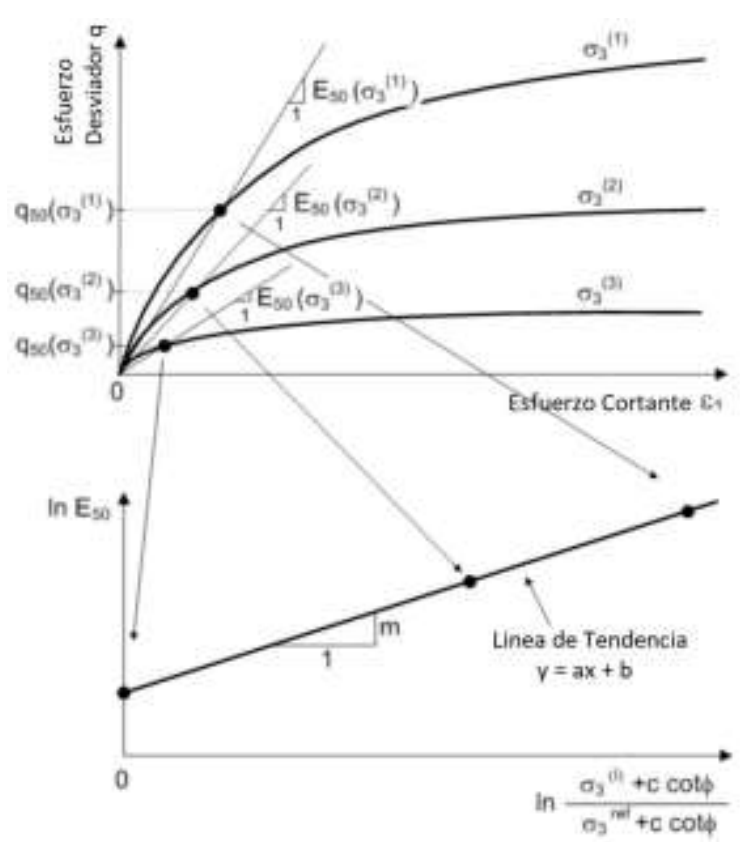

Figura 2. Determinación de m en función de los resultados de un ensayo triaxial

Donde:

$\sigma_{3}^{(1)}, \sigma_{3}^{(2)}, \sigma_{3}^{(3)}$

Curva Esfuerzo - Deformación del suelo para diferentes presiones de confinamiento.

Definido el parámetro $\mathrm{m}$ y E50 se procede a calcular $F_{50}^{r e f}$ mediante la siguiente expresión, la cual ha sido tomada directamente de los creadores de este modelo 1 [4, p. 2].

$$
E_{50}=E_{50}^{r e f}\left(\frac{c \cdot \cot \phi-\sigma_{3}}{\sigma_{\text {ref }+ \text { c.cot } \phi}}\right)^{m}
$$

El parámetro $E_{\text {eod }}^{r e f}$ si se cuenta con un ensayo edométrico se calcula directamente mediante:

$E_{\text {oed }}=\frac{2.3\left(1+e_{\text {ref }}\right)}{C c} \sigma_{\text {eod }}^{\text {ref }}$

$e_{\text {ref }}$ Representa a la relación de vacíos asociada a la presión de preconsolidación que los textos de mecánica de suelo normalmente la denominan $\sigma_{m}$, Cc es el índice de compresibilidad que se obtiene de un ensayo edométrico, definido $\mathrm{E}^{\text {oed }}$ se $\mathrm{lo}$ referencia a un estado de presiones mediante:

$$
E_{\text {oed }}=E_{\text {oed }}^{\text {ref }}\left(\frac{c \cdot \cos \phi-\frac{\sigma_{3}}{K_{o}^{n C}} \operatorname{sen} \sigma \phi}{c \cdot \cos \phi+\sigma_{\text {ref }} \cdot \operatorname{sen} o \phi}\right)^{m}
$$

El valor dęsé lo puede estimar mediante el índice de hinchamiento (Cs) del ensayo edométrico mediante la siguiente ecuación:

$$
C_{s} \approx \frac{2.3\left(1+e_{0}\right)(1+v)(1-2 v) \sigma_{r e f}}{(1-v) E_{u r}^{r e f}}
$$

El valor del coeficiente de poisson $v$ se lo puede definir directamente mediante el coeficiente de reposos del suelo $K_{n}^{\text {nnt }}$ también se puede definir en base a una relación elástica entre el módulo de Young que está definido por $E^{50}$ mediant $E_{5 \Theta}$ donde $G$ representa módulo de corte $E_{50}$

$$
\begin{aligned}
& K_{o}^{n c}=\frac{v}{1-v} \\
& E_{50}=2 G(1+v) \\
& E_{50}=\frac{8}{3} G
\end{aligned}
$$

Con el fin de garantizar cálculos realistas el módulo de volumen del agua debe ser alto en comparación con el módulo de volumen del esqueleto del suelo. Esta condición está suficientemente asegurada al requerir $v \leq 0.35[5$, p. 24].

El Ko no se calcula de la misma manera para todos los tipos del suelo para arcillas normalmente consolidadas ko y para suelos de granos gruesos sobre consolidados ko, esto según los propios autores de las formulaciones [6] y [7] respectivamente. Hay que tomar en consideración que Ko no puede ser mayor que 1 .

$\mathrm{k}_{0}=1-\operatorname{seno} \phi$

$\mathrm{k}_{0}=(1-\operatorname{sen} o \phi) O C R^{\mathrm{Sen} o \phi}$

Para suelos granulares $v_{u r}$ se obtiene directamente mediante Eo, si no se cuenta con ensayos cíclicos lo más práctico es emplear correlaciones para definir dichos parámetros. Uno de los parámetros se calcula mediante Go [2, p. 132], donde Vs es la velocidad de ondas de corte. El parámetro Eo para todos tipos de suelos para humedades naturales menores al 50\% según [8] se puede calcular mediante:

$$
\begin{aligned}
& \mathrm{E}_{0}=2\left(1+v_{u r}\right) G o \\
& G_{O}=\frac{a v_{s}^{3}}{s}
\end{aligned}
$$


$E_{0}=\frac{140}{e_{0}}\left(\frac{\sigma_{3}}{\sigma_{r A j}}\right)^{0.5} M P^{P} a$

Por lo tanto $G_{0}=G_{0}^{\text {ref }}\left(\frac{c \cdot \cot \phi-\sigma_{3}}{\sigma_{r e f+c . \cot \phi}}\right)^{m}$

El ángulo de dilatancia se puede obtenermediante [3, p. 107].

$\psi=0$ Para suelos normal yligeramente consolidados

$\psi=\frac{\phi}{6}$ Para suelos sobre consolidados

$\psi=\frac{\phi}{2}$ Para suelos fuertemente sobre consolidados

La interfaz por otro lado se emplea para simular la rigidez virtual que se genera entre el suelo y la cimentación y cuyo cálculo se lo realiza a partir de las siguientes ecuaciones [26, p.189]

$$
\begin{aligned}
& K n=\frac{E_{\text {oed }}}{l . . t v} \\
& K t=\frac{G i}{\text { L.t. }} \\
& \text { Gi. }=\text { R. Gsoil. } \\
& \text { Gsoil }=\frac{E}{2\left(2+v_{d}\right)}
\end{aligned}
$$

Donde:

$E_{\text {ouri }}:$ Es el módulo de elasticidad de ensayos edométricos

$v_{i} \quad$ : Coeficiente de Poisson de la interfaz, cuyo valor recomendado es de 0.45

$L \quad$ : es la longitud de análisis, que por default se le asigna $1 \mathrm{~m}$

to : Es el espesor virtual de la interfaz (Generalmente tiene un valor entre 0.01 0.1 , cuanto mayor sea la diferencia de rigidez entre el suelo y la estructura, menor será el valor)

$R \quad$ : Factor de reducción estructural cuyos valores son:

$\begin{array}{ll}\text { Arena/Acero } & =0.6-0.7 \\ \text { Arcilla/Acero } & =0.5 \\ \text { Arena/Hormigón } & =0.80-1.00 \\ \text { Arcilla/Hormigón } & =0.70-1.00\end{array}$

\section{Desarrollo}

La ubicación del proyecto está en las coordenadas:

Este: $779965.05 \mathrm{E}$

Sur: 9963229.87S.

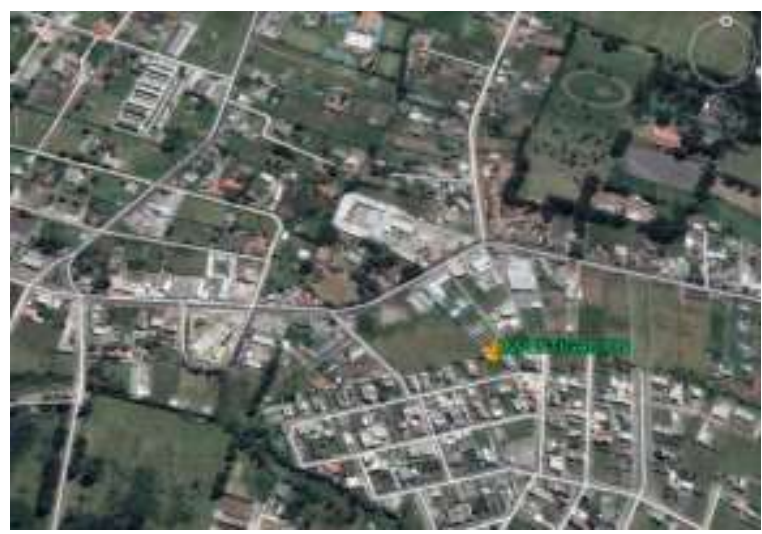

Figura 3. Ubicación de Google Earth del lugar de investigación

Se realizaron 5 sondeos SPT en los cuales se extrajeron muestras inalteradas para la ejecución de 4 ensayos triaxiales y un ensayo de consolidación. Además, se realizaron ensayos geofísicos como el VS30 y periodos de vibración natural del depósito por el método [9].

Nakamura propone un método para la estimación de características dinámicas de las capas superficiales utilizando Micro tremores en la superficie, siendo el micro tremor aquel movimiento registrado en el suelo de corto periodo accionado por fuerzas artificiales, en esta investigación se observó que el tremor horizontal se ve amplificado a través de la multireflexión de la onda $\mathrm{S}$ mientras que el tremor vertical es a través de la reflexión de la onda $\mathrm{P}$, dando como resultado que la relación de los espectros horizontales y verticales del micro tremor es una función de transferencia aproximada [9].

Los resultados de los ensayos triaxiales en términos efectivos son los siguientes: 
Tabla 3. Resultados ensayos triaxiales

\begin{tabular}{clllll}
\hline Pozo & $\begin{array}{l}\text { Prof. } \\
(\mathbf{m})\end{array}$ & $\begin{array}{l}\gamma \mathrm{h} / \gamma \mathbf{s a t} \\
\left(\mathrm{T} / \mathbf{m}^{3}\right)\end{array}$ & $\begin{array}{l}\gamma \mathrm{s} \\
\left(\mathrm{T} / \mathbf{m}^{3}\right)\end{array}$ & $\begin{array}{l}\text { c }(\mathbf{k g} / \\
\left.\mathbf{c m}^{2}\right)\end{array}$ \\
\hline Pz5 & $\begin{array}{l}0.5- \\
1.5\end{array}$ & 1.83 & 1.42 & 8.66 & 0.29 \\
\hline Pz5 & $\begin{array}{l}1.5- \\
2.5\end{array}$ & 1.83 & 1.34 & 7.03 & 0.21 \\
\hline Pz5 & $\begin{array}{l}2.5- \\
3.5\end{array}$ & 1.74 & 1.28 & 5.14 & 0.18 \\
\hline Pz4 & $\begin{array}{l}4.5- \\
5.5\end{array}$ & 1.79 & 1.31 & 6.11 & 0.177 \\
\hline
\end{tabular}

Los resultados del ensayo edométrico son los siguientes:

Tabla 4. Resultados ensayos de consolidación

\begin{tabular}{|c|c|c|c|c|}
\hline \multicolumn{5}{|c|}{ RESULTADOS } \\
\hline$\gamma \mathrm{h}=$ & 1.75 & $\begin{array}{l}\text { Presión } \\
\left(\mathrm{kg} / \mathrm{cm}^{2}\right)\end{array}$ & e1 & $\mathrm{K}(\mathrm{cm} / \mathrm{seg})$ \\
\hline$\gamma \mathrm{S}=$ & $\underline{1.37}$ & $\underline{0.51}$ & $\underline{0.551}$ & $1.18 \mathrm{E}-06$ \\
\hline $\mathrm{Cc}=$ & $\underline{0.134}$ & $\underline{1.02}$ & $\underline{0.536}$ & $\underline{3.93 \mathrm{E}-07}$ \\
\hline $\mathrm{Cr}=$ & $\underline{0.078}$ & 2.04 & $\underline{0.513}$ & $5.55 \mathrm{E}-07$ \\
\hline $\mathrm{Cs}=$ & $\underline{0.014}$ & 4.08 & 0.482 & $\underline{4.42 \mathrm{E}-07}$ \\
\hline $\mathrm{eo}=$ & 0.59 & 8.15 & 0.442 & $1.43 \mathrm{E}-07$ \\
\hline
\end{tabular}

$\sigma \mathrm{m}\left(\mathrm{kg} / \mathrm{cm}^{2}\right)=\underline{3.14}$

$\sigma 0\left(\mathrm{~kg} / \mathrm{cm}^{2}\right)=\underline{0.79}$

$\mathrm{OCR}=$

4

Para estimar el Vs30 se empleó el método combinado ReMi/MASW y cuyos valores se reportan a continuación:

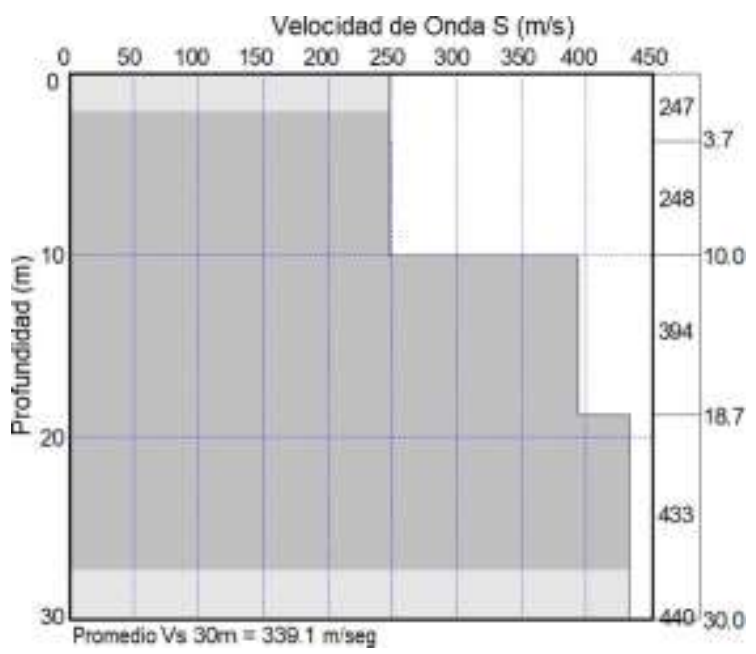

Figura 4. Valores de velocidades de ondas de corte Vs combinados
Se ha estimado un valor de $V_{\mathrm{s30}}=339 \mathrm{~m} / \mathrm{seg}$

Mientras que el cociente espectral H/V del método Nakamura ha estimado un valor de Perio$\mathrm{do}=0.978 \mathrm{~Hz}=1.022 \mathrm{seg}$.

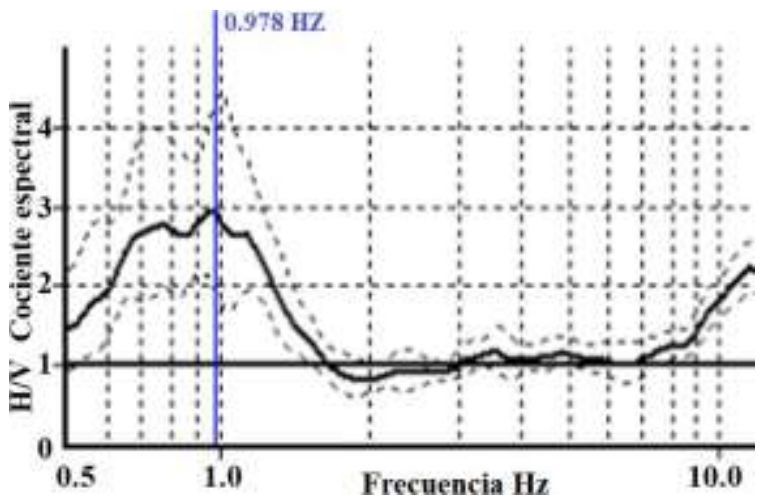

Figura 5. Espectro de cocientes espectrales H/V.

\subsection{Espectro de sitio}

\subsection{A partir de la NEC-15}

El análisis se desarrolla en el Cantón Quito, para una estructura de hormigón Armado, para una velocidad de ondas de corte de $339 \mathrm{~m} / \mathrm{seg}$, aplicando la [24] se tiene:

Tabla 5. Coeficientes sísmicos

\begin{tabular}{lccc}
\hline \multicolumn{4}{c}{ CARGA DE SISMO-NEC-SE-DC } \\
\hline Parámetro & Valor & Unidad \\
\hline Coeficiente $\mathrm{Ct}=$ & 0.055 & \\
\hline $\begin{array}{l}\text { Coeficiente para cálculo de Periodo } \\
\boldsymbol{\alpha}=\end{array}$ & 0.9 & & \\
\hline Periodo Natural de Vibración T1= & 1.24 & seg \\
\hline $\begin{array}{l}\text { Periodo Natural de Vibración } \\
\text { Máxima T2 }=\end{array}$ & 1.62 & seg \\
\hline Factor de importancia I= & 1 & \\
\hline Factor de reducción de respuesta $\mathrm{R}=$ & 8 & \\
\hline $\begin{array}{l}\text { Relación de amplificación espectral } \\
\mathrm{n}=\end{array}$ & 2.48 & \\
\hline Zona Sísmica $=$ & $\mathrm{V}$ & ALTA \\
\hline Factor de Zona $\mathrm{Z}=$ & 0.4 & \\
\hline Tipo de suelo $=$ & $\mathrm{D}$ & \\
\hline Factor de sitio Fa $=$ & 1.2 & \\
\hline Factor de Sitio Fd= & 1.19 & \\
\hline
\end{tabular}




\begin{tabular}{|c|c|c|}
\hline Parámetro & Valor & Unidad \\
\hline $\begin{array}{l}\text { Factor comportamiento no lineal } \\
\text { Fs }=\end{array}$ & 1.28 & \\
\hline Periodo $\mathrm{Tc}=$ & 0.70 & \\
\hline Factor $r=$ & 1 & \\
\hline Periodo $\mathrm{TL}=$ & 2.86 & \\
\hline Periodo Modelo $($ Tmod $)=$ & 1.2445 & \\
\hline Aceleración espectral $\mathrm{Sa}=$ & 0.67 & $\mathrm{~g}$ \\
\hline
\end{tabular}

Factor irregularidad en Planta $\Phi \mathbf{p}=\quad \underline{1}$

Factor irregularidad en elevación $\quad 1$ $\Phi \mathrm{E}=$

\begin{tabular}{lc}
\hline Factor de reducción $(\mathrm{f})=$ & 0.1250 \\
\hline Factor $\mathrm{k}$ & 1.37 \\
\hline
\end{tabular}

Los coeficientes sísmicos de la tabla 3, permiten realizar el grafico del espectro, el cual se encuentra representado en la gráfica 2 .

\subsection{A partir del segmento de falla de Quito}

La falla de Quito se divide en ocho segmentos, se ha optado por estimar los espectros a partir de la data base del PEER [10].

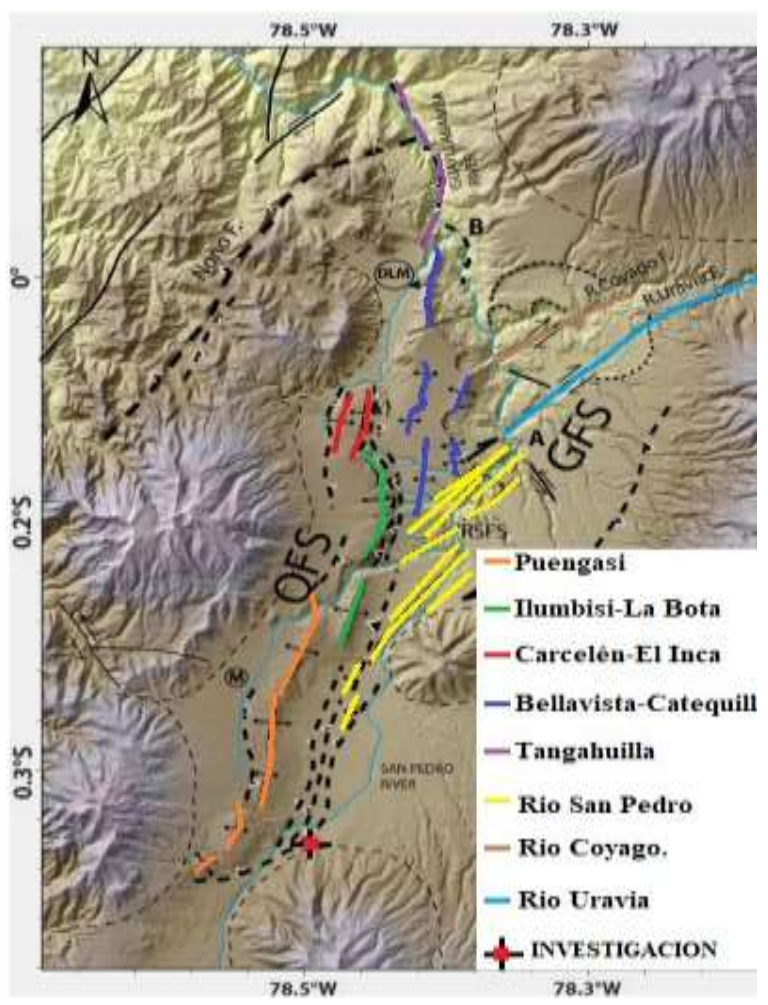

Ztor Profundidad hasta el borde superior del plano de ruptura en $\mathrm{Km}$

$W \quad$ Ancho de la falla en $\mathrm{Km}$

Dip $\delta \quad$ Angulo de buzamiento promedio del plano de ruptura en grados

Rake $\lambda$ Angulo entre la dirección de deslizamiento en el plano de falla y la orientación de la falla en la superficie

VS30 Velocidades de ondas de corte a $30 \mathrm{~m}$ de profundidad $\mathrm{m} / \mathrm{seg}$

Z $\quad$ Profundidad a la que la velocidad de la onda de corte es $\mathrm{Vs}=1.0 \mathrm{Km} / \mathrm{seg}$

$Z_{2.5} \quad$ Profundidad a la que la velocidad de la onda de corte es $\mathrm{Vs}=2.5 \mathrm{Km} / \mathrm{seg}$

Profundidad hipocentral del sismo en $\mathrm{km}$

La referencia de las fórmulas empleadas se las puede encontrar en [12]:

Figura 6. Segmentos de falla de Quito, modificado de [11] 
$\dot{N}_{M i n}$

$=\frac{M o \cdot(d-\beta)\left[e^{-\beta(\text { Mmen })}-e^{-\beta(M \max )}\right]}{\beta\left[e^{-\beta(M \max )} M o_{\max }-e^{-\beta(M \min )} M o_{\max }\right]}$

$N(m)=N_{M n}\left[\frac{e^{-\dot{\beta}(m)}-e^{-\beta(M \max )}}{e^{-\beta(M m t n)}-e^{-\beta(M a x)}}\right]$

$d=1.5 \ln (10)$

$=3.454$

$\operatorname{Tr}$

$=\frac{1}{N_{(m)}^{*}}$

$\beta=1.47$

El valor de $\beta$, representa al parámetro de relación Gutenberg-Richter, defino por [13].

La tasa de acumulada de sismos con GR Modificada se aprecia en la siguiente gráfica:

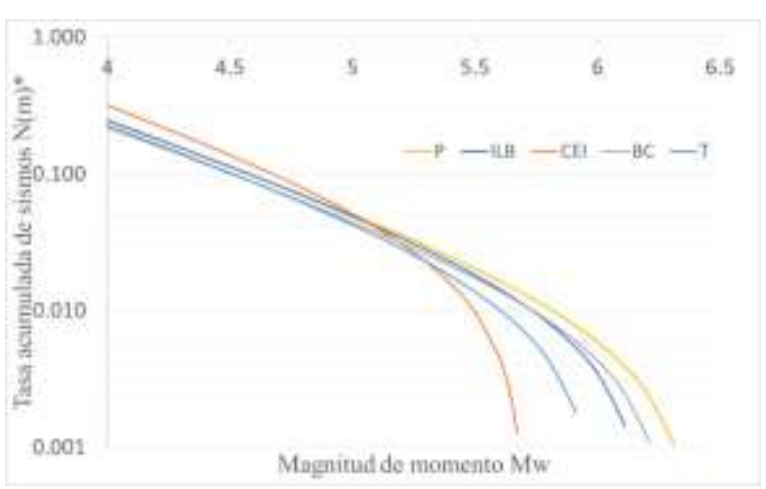

Figura 7. Tasa acumulada de sismos mediante GR modificada

Según [14], se debe asumir que el hipocentro se encuentra en el centroide del plano.

Los parámetros que se indican a continuación se pueden determinar de acuerdo con [15] y [16].

$Z_{\text {HYP }}=$

$(5.63+0.68 M w \quad$ fallas tipo Strike slip

$11.24+0.2 \mathrm{Mw} f$.que no son strike slip

$7.08+0.61 M w \quad$ fallas generales
$W=\left\{\begin{array}{lr}10^{-0.76+0.27 M w} & \text { fallas tipo Strike slip } \\ 10^{-1.61+0.41 M w} & \text { f.que no son strike slip } \\ 10^{-1.14+0.35 M w} & \text { fallas generales }\end{array}\right.$

$Z_{T O R}=\max \left[\left(Z_{H Y P}-0.6 W x \operatorname{seno} \delta\right), 0\right]$

Sabiendo que se ha asumido un ángulo $\alpha=-50^{\circ} \mathrm{y}$ que se encuentra entre $-180^{\circ} \leq \alpha<0^{\circ}$, la distancia $\mathrm{Rx}$ se calcula como:

$R x=-R_{J B} x \operatorname{seno\alpha }$

La distancia Rrup para ángulos $\delta \neq 90^{\circ}$, se debe calcular como:

$R_{R U P}=\sqrt{\left(R_{R U P}\right)^{2}+R y^{2}}$

$R_{R U P}^{\prime}=\sqrt{R x^{2}+Z_{T O R}^{2}}$

$R_{\text {RUP }}{ }^{\prime}=R x \operatorname{Seno} \delta+Z_{\text {TOR }} \operatorname{Cos} \delta$

$R_{\text {RUP }}$

$=\sqrt{(R x-W \operatorname{Cos} \delta)^{2}+\left(Z_{T O R}-W \operatorname{Seno} \delta\right)^{2}}$

sí $R x^{2}<+Z_{T O R} \tan \delta$

sí $Z_{T O R} \tan \delta \leq R x \leq Z_{\text {TOR }} \tan \delta+W \sec \delta$

sí $R x>Z_{\text {TOR }} \tan \delta+W \sec \delta$

$R y=\left\{\begin{array}{lr}0 & \text { para } \alpha=\mp 90^{\circ} \\ R_{J B} & \text { para } \alpha=0^{\circ} \circ \mp 180^{\circ} \\ |R x \cdot \cot \alpha| & \text { para cual quier otro }\end{array}\right.$

Según [16], Ry0 solo se debe usar para sitios en el lado HW (Hanging-wall site), en nuestro caso no aplica y el valor Ryo=0. El tipo de falla de Quito es Dips-Slip es decir de movimientovertical.

Z1.0 y Z2.0 se calcula de acuerdo a la siguiente ecuación: 
$Z 1.0=\left\{\begin{array}{lc}e^{6.745} & V s_{30}<180 \mathrm{~m} / \mathrm{s} \\ e^{\left(6.745-1.35 \ln \frac{V s}{180}\right)} & 180 \leq V s_{30} \leq \frac{500 m}{s}(49) \\ e^{5.394-4.48 \ln \frac{V s 30}{500}} & V s_{30}>500 m / s\end{array}\right.$

$Z_{2.5}=519+3.595 Z_{1.0}$

Z1.0: Profundidad a la que la velocidad de la onda de corte es Vs=1.0Km/seg

Z2.5: Profundidad a la que la velocidad de la onda de corte es Vs=2.5Km/seg

El resumen de los valores para encontrar el espectro de respuesta, en base a los modelos de atenuación de [17], [14] y [18],son:

Tabla 6. Resumen para definir el espectro de Puengasí

\begin{tabular}{llllll}
\hline Segmento & $\mathbf{P}$ & ILB & CEI & BC & T \\
\hline Tipo & Inversa & Inversa & Inversa & Inversa & Inversa \\
Longitud $(\mathrm{km})$ & 22 & 15 & 7 & 17.5 & 12 \\
Área $\left(\mathrm{km}^{2}\right)$ & 259 & 176 & 82 & 191 & 108 \\
Mw & 6.23 & 6.06 & 5.65 & 6.14 & 5.9 \\
RJB $(\mathrm{km})$ & 5.36 & 10.52 & 22.87 & 18.25 & 37.86 \\
Ángulo $\alpha$ & -50.00 & -50.00 & -50.00 & -50.00 & -50.00 \\
Zhyp $(\mathrm{km})$ & 12.49 & 12.45 & 12.37 & 12.47 & 12.42 \\
W (km) & 8.80 & 7.49 & 5.09 & 8.08 & 6.44 \\
Rx $(\mathrm{km})$ & -4.11 & -8.06 & -17.52 & -13.98 & -29.00 \\
Buzamiento $\delta^{\circ}$ & 55 & 55 & 55 & 55 & 55 \\
ZTOR $(\mathrm{km})$ & 8.16 & 8.77 & 9.87 & 8.50 & 9.25 \\
R RUP' $(\mathrm{km})$ & 9.14 & 11.91 & 20.11 & 16.36 & 30.44 \\
Ryo (km) & 3.45 & 6.76 & 14.70 & 11.73 & 24.34 \\
R RUP $(\mathrm{km})$ & 9.77 & 13.70 & 24.91 & 20.13 & 38.97 \\
\hline
\end{tabular}

El parámetro épsilon $\varepsilon$, se utiliza para definir el número de desviaciones estándar respecto a la mediana representada por un nivel de movimiento del suelo en particular [19].

Por ejemplo, el espectro de percentil 84 es un espectro donde los niveles de movimiento del suelo son una desviación estándar por encima de la mediana en todos los periodos espectrales y el valor de épsilon $\varepsilon=1$.

Ingresando todos los parámetros calculados del segmento de falla Puengasí, en la plataforma [10].

\begin{tabular}{|c|c|c|}
\hline \multicolumn{2}{|c|}{ NGA-West2 GMM } & Weight? \\
\hline \multicolumn{2}{|c|}{ Abrahamson-Silva-Kamai '14 } & 1.0 \\
\hline \multicolumn{2}{|c|}{ Boore-Stewart-Seyhan-Atkinson '14 } & '14 1.0 \\
\hline \multicolumn{2}{|c|}{$\checkmark$ Campbell-Bozorgnia '14 } & 1.0 \\
\hline \multicolumn{2}{|c|}{$\checkmark$ Chiou-Youngs '14 } & 1.0 \\
\hline \multicolumn{2}{|l|}{ Idriss '14 } & 1.0 \\
\hline \multicolumn{3}{|c|}{ Damping Ratio : $5 \% \mathbf{v}$} \\
\hline Region & \multicolumn{2}{|l|}{ : Global/California } \\
\hline Fault Type & \multicolumn{2}{|l|}{ : Reverse/Oblique $\mathbf{V}$} \\
\hline Magnitude & \multicolumn{2}{|l|}{6.23} \\
\hline Rrup (km) & \multicolumn{2}{|l|}{9.14} \\
\hline $\mathrm{Rx}(\mathrm{km})$ & \multicolumn{2}{|l|}{-4.11} \\
\hline Ry0 (km) & \multicolumn{2}{|l|}{0.0} \\
\hline $\operatorname{Rjb}(\mathrm{km})$ & \multicolumn{2}{|l|}{ : 5.36} \\
\hline Ztor $(\mathrm{km})$ & \multicolumn{2}{|l|}{ : 11.66} \\
\hline Width $(\mathrm{km})$ & \multicolumn{2}{|l|}{ : 8.8} \\
\hline DIP (deg) & \multicolumn{2}{|l|}{55.0} \\
\hline Vs30 (m/s) & \multicolumn{2}{|l|}{339.0} \\
\hline Vs30 type & \multicolumn{2}{|l|}{ : Measured $\boldsymbol{V}$} \\
\hline $\mathrm{Z} 1.0(\mathrm{~km})$ & 0.36 & Calc Defaults \\
\hline $\mathrm{Z2.5}(\mathrm{km})$ & \multicolumn{2}{|l|}{ : 1.82} \\
\hline Zhyp (km) & \multicolumn{2}{|l|}{ : 12.49} \\
\hline \multicolumn{3}{|c|}{ Hanging Wall : Y Yes $\bullet$ No } \\
\hline Epsilon & \multicolumn{2}{|l|}{ : 1.0} \\
\hline GMM Average & \multicolumn{2}{|c|}{ : Geometric Arithmetic } \\
\hline
\end{tabular}

Figura 8. Parámetros cargados en la plataforma PEER, para la falla de Puengasí

Se emplea un percentil 84 es un espectro donde los niveles de movimiento del suelo son una desviación estándar por encima de la mediana en todos los periodos espectrales y el valor de épsilon $\varepsilon=1$. En el siguiente gráfico se presentan los espectros de cada falla junto con el NEC-SE-DS- 15 .



Figura 9. Todos los espectros comparando con la NEC-SE-DS [20] 
ParaelanálisisdeISE, seemplealosespectrosdelaNECSE-DS, 2015 y de Puengasí, ya que son los más críticos.

\subsection{Características de la edificación}

La dimensión en planta de la estructura es de 20x23m, además cuenta con volados de $2.20 \mathrm{~m}$ y $2.50 \mathrm{~m}$.

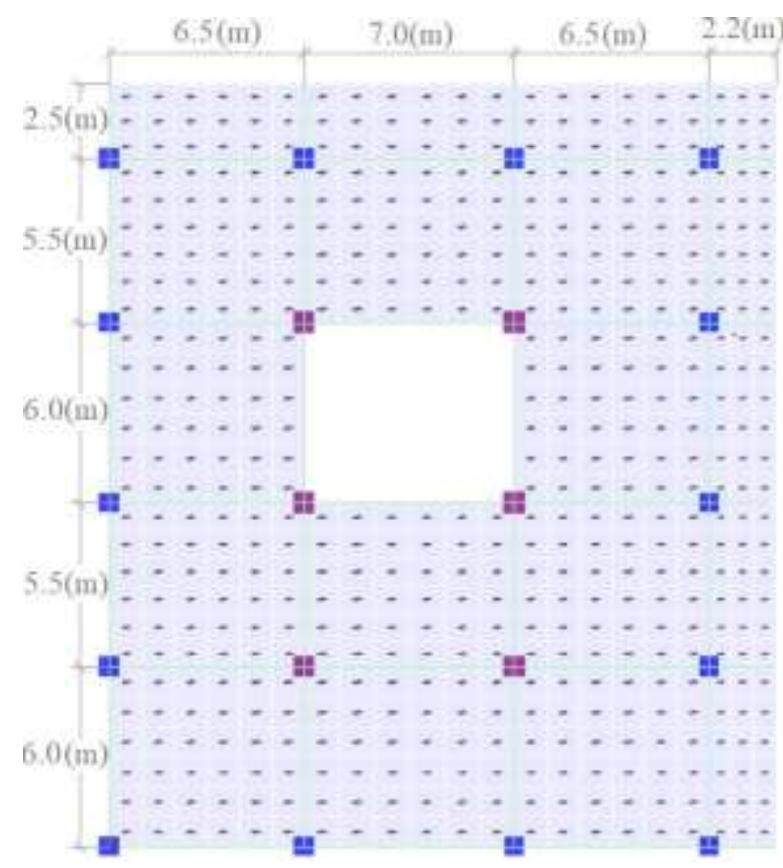

Figura 10. Dimensiones en planta

La edificación es de 8 niveles más un subsuelo, la altura de entre pisos es de $3.24 \mathrm{~m}$ con excepción de la tapagrada cuya altura es de $3.06 \mathrm{~m}$.

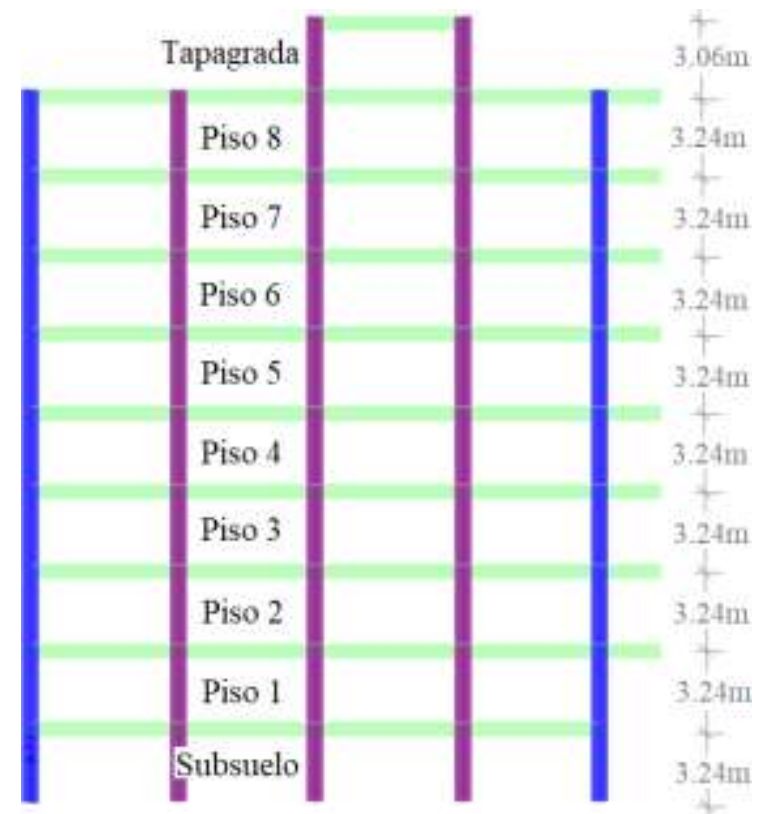

Es una estructura de hormigón armado con una resistencia a la compresión simple del hormigón $\mathrm{f}^{\prime} \mathrm{c}=40 \mathrm{MPa}$ y un acero de refuerzo con una resistencia a la fluencia en barras de $F y=420 \mathrm{MPa}$.

Tabla 7. Características del hormigón y acero de refuerzo en barras

\begin{tabular}{|c|c|c|c|}
\hline Descripción & Hormigón & Acero & Unid. \\
\hline $\begin{array}{l}\text { Peso por } \\
\text { volumen }\end{array}$ & 23.5631 & 76.9729 & $\mathrm{KN} / \mathrm{m}^{3}$ \\
\hline $\begin{array}{l}\text { Masa por } \\
\text { volumen }\end{array}$ & 2402.77 & 7849.074 & $\mathrm{Kg} / \mathrm{m}^{3}$ \\
\hline $\begin{array}{l}\text { Módulo de } \\
\text { elasticidad }\end{array}$ & 29725.41 & 199947.98 & $\mathrm{MPa}$ \\
\hline Coef. De Poisson & 0.2 & - & - \\
\hline $\begin{array}{l}\text { Coef. Expa. } \\
\text { Termal }\end{array}$ & 0.0000099 & 0.0000117 & $\mathrm{I} / \mathrm{C}$ \\
\hline $\begin{array}{l}\text { Módulo de corte } \\
\text { G }\end{array}$ & 12385.59 & - & $\mathrm{MPa}$ \\
\hline $\mathrm{Fe}$ & 40 & - & $\mathrm{MPa}$ \\
\hline Fy & - & 420 & $\mathrm{MPa}$ \\
\hline $\mathrm{Fu}$ & - & 620 & $\mathrm{MPa}$ \\
\hline Fye & - & 455 & $\mathrm{MPa}$ \\
\hline Fue & - & 683 & $\mathrm{MPa}$ \\
\hline
\end{tabular}

En la estructura se definieron columnas de $650 \times 650 \mathrm{~mm}$ (bordes y esquineras) y de 700x7000mm (centrales), vigas de 500x650mm, una cimentación de $500 \mathrm{~mm}$, losa de entrepiso maciza de $180 \mathrm{~mm}$ y muros de sótano de $250 \mathrm{~m}$. Cada uno de los elementos con su respectivo valor de inercias agrietadas 0.8 columnas, 0.5 vigas, 0.5 losas y 0.6 para los sótanos [21, p. 54].

\subsection{Método de la subestructura}

Modelar un edificio con sótanos según la [22, p. 6.7], tiene varias alternativas. 


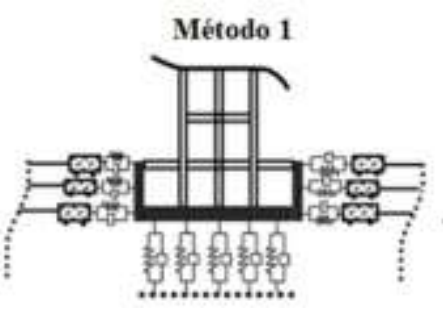

Método 2
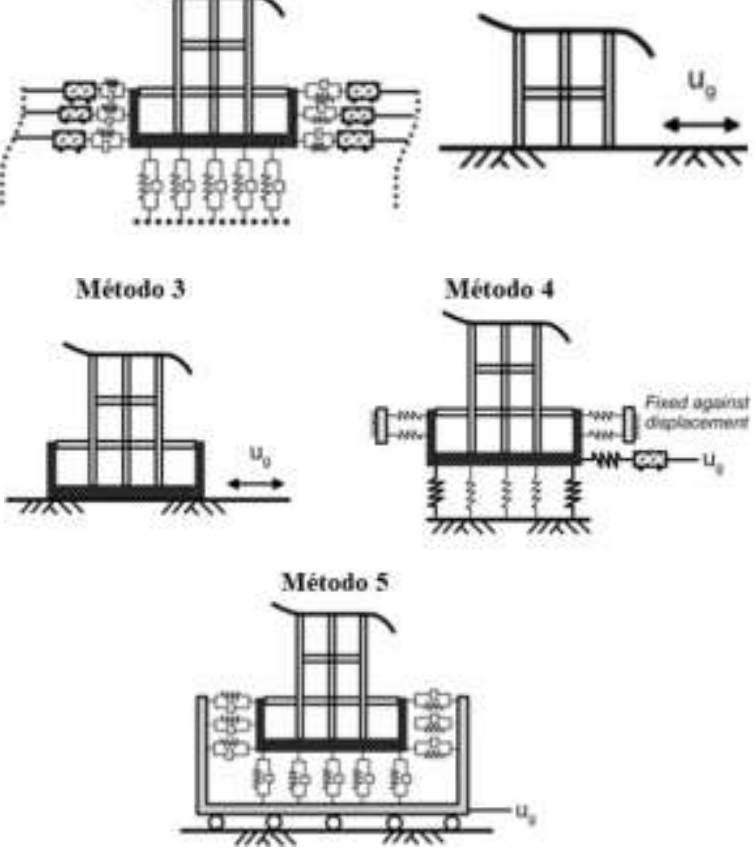

Figura 12. Métodos para modelar estructuras con sótanos

El método 2 (Base rígida), es el más empleado y el que no involucra un análisis de ISE y este método será empleado para comparar los resultados de los otros modelos. El método 4, aunque no contempla todos los parámetros del suelo representa un modelo avanzado ya que involucra la introducción de rigideces mediante las funciones de impedancia que representan al suelo, tanto en la cimentación como en los sótanos.

\subsection{Base rígida-Modelo $\mathrm{A}$}

Se ingresa los espectros de sitio multiplicando por el factor de reducción de 0.125 de la tabla 5 .

En la norma Ecuatoriana de la Construcción-Capitulo de Peligro Sísmico y Diseño Simoresistente [21], solicita que los espectros de respuesta deben tener al menos un $85 \%$ de fuerza basal al comparar con el sismo estático, dicho en otras palabras, los espectros ingresados han sido calibrados para cumplir dicha especificación.

Los periodos de vibración son los siguientes:
Tabla 8. Modos de vibración de la estructura con base rígida

\begin{tabular}{|c|c|c|}
\hline Caso & Modo & Periodo(seg) \\
\hline Modal & 1 & 1.06 \\
\hline Modal & 2 & 0.98 \\
\hline Modal & 3 & 0.89 \\
\hline Modal & 4 & $\underline{0.33}$ \\
\hline Modal & 5 & $\underline{0.31}$ \\
\hline Modal & $\underline{6}$ & $\underline{0.28}$ \\
\hline Modal & 7 & $\underline{0.18}$ \\
\hline Modal & $\underline{8}$ & $\underline{0.17}$ \\
\hline Modal & 9 & 0.16 \\
\hline Modal & $\underline{10}$ & $\underline{0.12}$ \\
\hline Modal & $\underline{11}$ & $\underline{0.11}$ \\
\hline Modal & $\underline{12}$ & $\underline{0.10}$ \\
\hline Modal & 13 & $\underline{0.08}$ \\
\hline Modal & $\underline{14}$ & $\underline{0.08}$ \\
\hline Modal & $\underline{15}$ & $\underline{0.07}$ \\
\hline Modal & 16 & $\underline{0.07}$ \\
\hline Modal & 17 & $\underline{0.07}$ \\
\hline Modal & 18 & $\underline{0.06}$ \\
\hline Modal & 19 & $\underline{0.05}$ \\
\hline Modal & 20 & 0.05 \\
\hline Modal & 21 & $\underline{0.04}$ \\
\hline Modal & $\underline{22}$ & $\underline{0.04}$ \\
\hline Modal & 23 & $\underline{0.03}$ \\
\hline Modal & 24 & $\underline{0.02}$ \\
\hline
\end{tabular}

Las derivas máximas en $\mathrm{X}$ e $\mathrm{Y}$ son las siguientes.



Figura 13. Derivas máximas del Modelo A, con Espectro NEC 




Figura 14. Derivas máximas del Modelo A, con Espectro Puengasí

Según [21, p. 69] las derivas finales se calculan como:

$$
\Delta m=0.75 x R x \Delta_{R}
$$

Para el espectro NEC se tiene:

$\Delta \mathrm{x}=0.01136$

$\Delta y=0.00981$

\section{Para el espectro Puengasí se tiene:}

$\Delta \mathrm{x}=0.01102$

$\Delta y=0.0090$

\subsection{ISE con funciones de impedancia-Modelo B}

Lo que trata este modelo es el de representar mediante un sistema lineal equivalente el comportamiento no lineal del suelo asociados a unas deformaciones aceptables, el punto de partida por lo tanto es estimar Gmax a partir de mediciones de campo como el Vs30 [2, p. 132].

$$
G_{\max }=\frac{\rho V_{s}^{2}}{g}
$$

\section{Donde:}

$\begin{array}{ll}G_{\max } & \text { Módulo de corte estimado } \\ \rho & \text { Densidades del suelo } \\ V_{s} & \text { Velocidades de ondas de corte }\end{array}$

De los ensayos triaxiales y las mediciones en campo del peso unitario del suelo, está en el orden de $1.80 \mathrm{~T} / \mathrm{m} 3$ y el ensayo Vs tiene un valor de $248 \mathrm{~m} /$ seg, para de una profundidad de $-10.0 \mathrm{~m}$ (ver figura 3).

$$
G_{\max }=\frac{1.80^{\mathrm{T}} / \mathrm{m}^{3} \times(248 \mathrm{~m} / \mathrm{s})^{2}}{9.81 \mathrm{~m} / \mathrm{s}^{2}}=110.76 \mathrm{MPa}
$$

Según ASCE 41 [2, p. 132], el valor de G/Gmax se puede obtener de la siguiente tabla:

Tabla 9. Tabla de Módulo cortante efectivo G/Gmax

\begin{tabular}{ccccc}
\hline \multicolumn{5}{c}{ Pico de aceleraciones efectivas PGA (a) } \\
\hline Tipo & PGA=0 & PGA=0.1 & PGA=0.4 & PGA=0.8 \\
\hline A & 1 & 1 & 1 & 1 \\
B & 1 & 1 & 0.95 & 0.9 \\
C & 1 & 0.95 & 0.75 & 0.6 \\
D & 1 & 0.9 & 0.5 & 0.1 \\
E & 1 & 0.6 & 0.05 & (b) \\
F & (b) & (b) & (b) & (b) \\
\hline
\end{tabular}

(a) Use interpolación lineal para valores intermedios de PGA

(b) Se realizarán investigaciones geotécnicas específicas del sitio y análisis dinámicos de la respuesta del sitio

A este perfil de suelo se lo clasifica como Tipo D y un $\mathrm{PGA}=0.4$, donde Gsec es:

$$
\begin{aligned}
G_{\text {sec }}=0.5 \times G_{\max } & =0.5 \times 110.76 \mathrm{MPa} \\
& =55.38 \mathrm{MPa}
\end{aligned}
$$

El procedimiento para el cálculo de las rigideces dinámicas y amortiguamiento es la siguiente:

- Se calcula la rigidez dinámica para x, y, z y sus componentes rotacionales

- Se calcula los factores de corrección por embebido para $\mathrm{x}, \mathrm{y}, \mathrm{z}$ y sus componentes rotacionales $\eta$ 
- Se calcula los modificadores de rigidez dinámica por radiación de ondas para $\mathrm{x}, \mathrm{y}, \mathrm{z}$ y sus componentes rotacionales $\alpha$

- Se calcula los modificadores de rigidez dinámica por radiación de ondas para $\mathrm{x}, \mathrm{y}, \mathrm{z}$ y sus componentes rotacionales $\beta$

- Se calcula la componente de rigidez dinámica $\mathrm{K}_{\mathrm{emb}}=\mathrm{Kx} \eta \mathrm{x} \alpha$ para $\mathrm{x}, \mathrm{y}, \mathrm{z}$ y sus componentes rotacionales

- Se calcula la componente de amortiguamiento $C=\frac{K e m b x \beta}{\omega}$ para $\mathrm{x}, \mathrm{y}$ e $\mathrm{z}$ y sus componentes rotacionales ( es la frecuencia de vibración de la estructura).

- Se divide Kemb y C para el área de cimentación.

Esto involucra distancias entre los bordes de la cimentación donde la rigidez toma valores más altos que al compararlos con las esquinas y centro.

Tabla 10. Resumen de rigideces. Fuente: Los Autores

\begin{tabular}{ccccc}
\hline \multirow{4}{*}{ Rigidez } & \multicolumn{3}{c}{$\begin{array}{c}\text { Rigidez (KN/m3)/Direc- } \\
\text { ción local }\end{array}$} & \\
\cline { 2 - 4 } & 1 & 2 & 3 & \\
\hline KB & 7058 & 6973 & 35040 & $\begin{array}{c}\text { Dimensión } \\
\text { corta }\end{array}$ \\
\hline KCENTRO & 7058 & 6973 & 10160 & Centro \\
\hline KESQ & 7058 & 6973 & 33210 & Centro \\
\hline KL & 7058 & 6973 & 31370 & $\begin{array}{c}\text { Dimensión } \\
\text { larga }\end{array}$ \\
\hline $\begin{array}{c}\text { KMURO-BA- } \\
\text { SE }\end{array}$ & 0 & 0 & 20730 & $\begin{array}{c}\text { Dimensión } \\
\text { corta }\end{array}$ \\
\hline $\begin{array}{c}\text { KMU- } \\
\text { RO-LARGO }\end{array}$ & 0 & 0 & 17810 & $\begin{array}{c}\text { Dimensión } \\
\text { larga }\end{array}$ \\
\hline
\end{tabular}

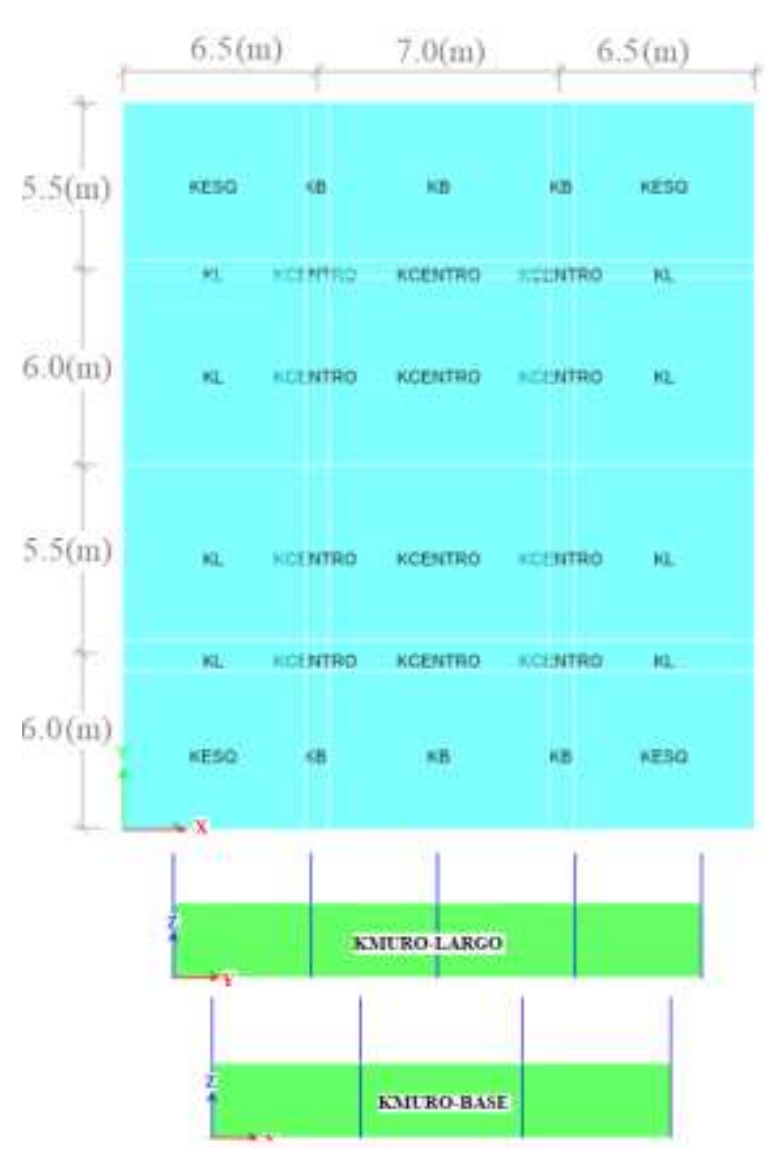

Figura 15. Asignación de rigideces

$$
\begin{aligned}
& \text { Rex }=5.75 \mathrm{~m} \\
& \text { Rey }=5.00 \mathrm{~m}
\end{aligned}
$$

Los periodos de vibración asociados a una estructura con base flexible son los siguientes:

Tabla 11. Modos de vibración de la estructura con base flexible

\begin{tabular}{ccc}
\hline Caso & Modo & Periodo (seg) \\
\hline Modal & 1 & 1.55 \\
Modal & 2 & 1.43 \\
\hline Modal & 3 & 1.24 \\
\hline Modal & 4 & 0.46 \\
\hline Modal & 5 & 0.43 \\
\hline Modal & 6 & 0.39 \\
\hline
\end{tabular}




\begin{tabular}{lcc}
\hline Caso & Modo & Periodo (seg) \\
\hline Modal & 7 & 0.26 \\
\hline Modal & 8 & 0.24 \\
\hline Modal & 9 & 0.22 \\
\hline Modal & 10 & 0.17 \\
\hline Modal & 11 & 0.16 \\
\hline Modal & 12 & 0.15 \\
\hline Modal & 13 & 0.12 \\
\hline Modal & 14 & 0.12 \\
\hline Modal & 15 & 0.10 \\
\hline Modal & $\underline{16}$ & $\underline{0.10}$ \\
\hline Modal & 17 & 0.09 \\
\hline Modal & 18 & 0.09 \\
\cline { 1 - 2 } Modal & 19 & 0.08 \\
\hline Modal & 20 & 0.08 \\
\hline Modal & 21 & 0.07 \\
\hline Modal & $\underline{22}$ & $\underline{0.06}$ \\
\hline Modal & 23 & 0.04 \\
\hline Modal & $\underline{24}$ & $\underline{0.03}$ \\
\hline & &
\end{tabular}

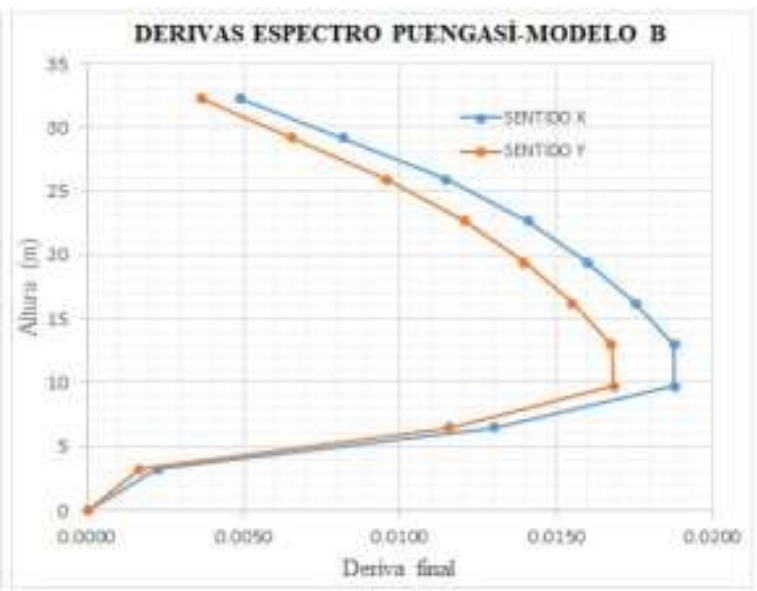

Figura 17. Derivas máximas base flexible con Espectro Puengasí

Para el espectro NEC:

$\Delta \mathrm{x}=0.020$

$\Delta \mathrm{y}=0.0176$

Para el espectro Puengasí:

$\Delta \mathrm{x}=0.0188$

$\Delta \mathrm{y}=0.0168$

Las derivas máximas en $\mathrm{X}$ e Y son las siguientes.

Valores inferiores a 0.02 que reporta [21].

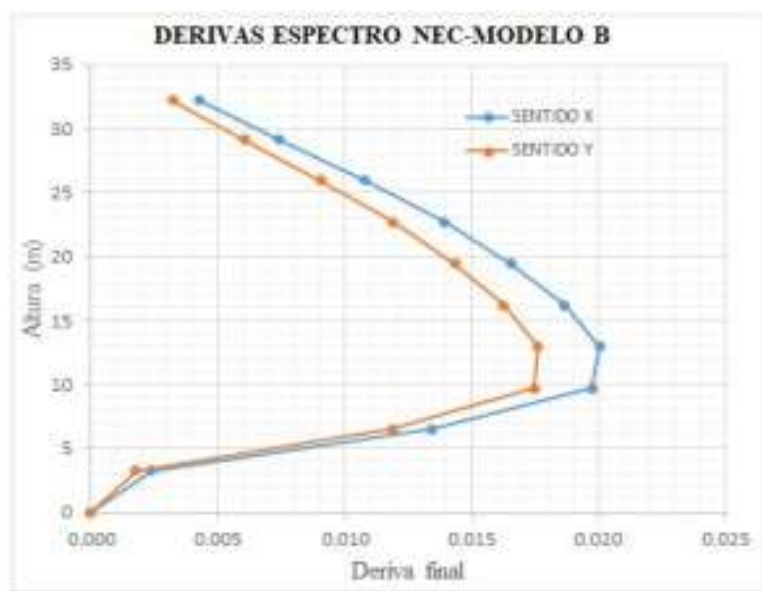

Figura 16. Derivas máximas base flexible con Espectro NEC

\subsection{Método directo de ISE-Modelo C}

El modelo directo representa tanto al suelo como la estructura directamente en la ISE, para realizar este análisis es necesario el empleo de elementos finitos. Para emplear este método es necesario emplear un modelo de rotura adecuado del suelo en donde se introduzcan todos los parámetros adecuados de la representación de la no linealidad del suelo. Para este modelo, se emplea la ecuación de movimiento completa $p(t),[21]$

$M \ddot{U}+C \dot{U}+K U=p(t)$

\subsection{Modelo HSSMALL}

Se presenta los cálculos de triaxial para el modelo HSSMALL. Se debe considerar que existen 
parámetros por default en este modelo por ejemplo que es la presión de referencia que se asigna $100 \mathrm{KPa}=1.02 \mathrm{~kg} / \mathrm{cm} 2$ que no es más que la presión atmosférica. Otro parámetro es la relación de falla $\mathrm{Rf}$ que es igual a0.9.

Para el cálculo de $\mathrm{E}_{50}^{\mathrm{ref}}$ se traza la línea qf, para posterior encontrar q al $50 \%$ de qf, se traza la recta que cruza q50\% y se determina la pendiente de la recta que resulta ser. Posterior es necesario realizar la curva de dependencia $E_{50}$ vs los estados de esfuerzos. Este procedimiento servirá para tratar de definir el valor de $\mathrm{m}$ (dependencia de la rigidez al estado de esfuerzos).

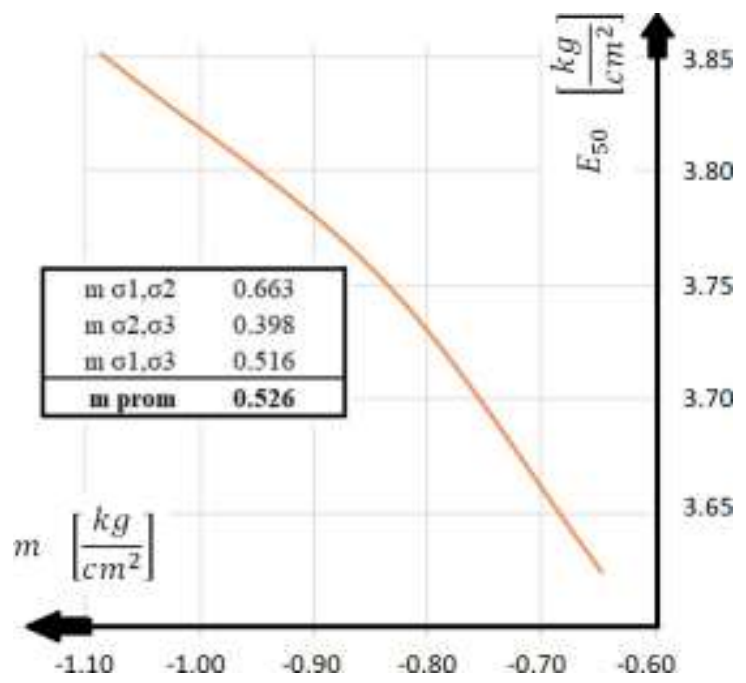

Figura 18. Dependencia $E_{50}$ con los estados de esfuerzo

Se tiene tres valores de m para cada combinación de los esfuerzos efectivos, cada uno de los cuales es diferente, debido a que este suelo no cumple como una recta.

El valor m esta entre 0.65 a 0.8 , por otro lado, de la gráfica 2 se tiene varios valores, siendo el de 0.66 el que más se ajusta.

$\mathrm{E}_{50}^{\mathrm{ref}}$ se realiza para cada presión de confinamien-

to $\sigma_{3}$.

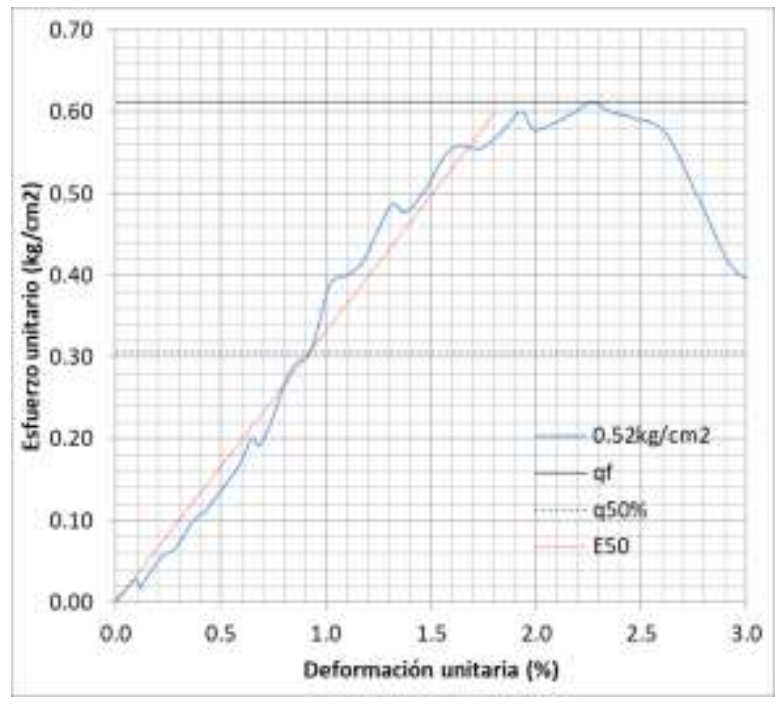

Figura 19. $E_{50}^{r e f}$ para una presión de confinamiento

$$
\sigma_{3}=0.52 \mathrm{~kg} / \mathrm{cm}^{2}
$$

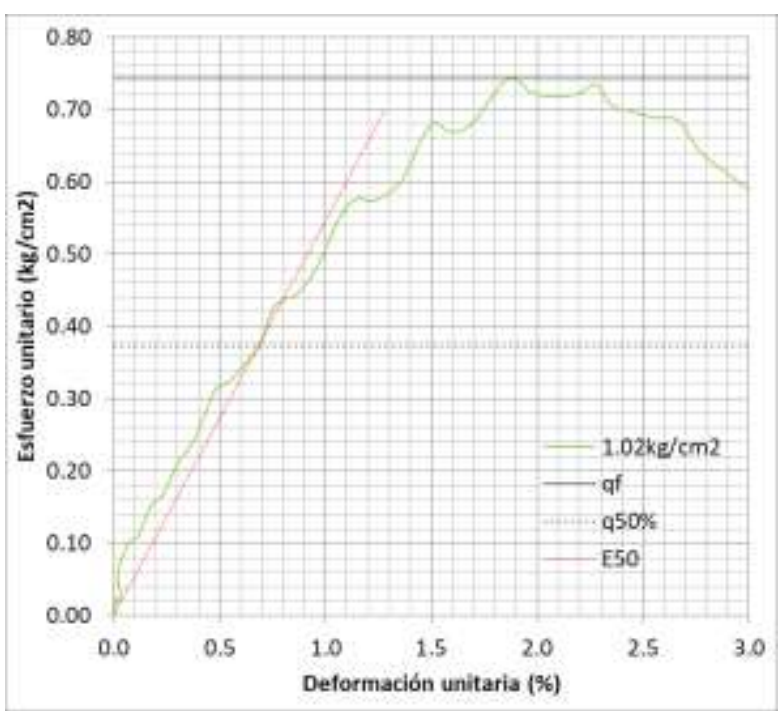

Figura 20. $\mathrm{E}_{50}^{\mathrm{ref}}$ para una presión de confinamiento

$$
\sigma_{3}=0.50 \mathrm{~kg} / \mathrm{cm}^{2}
$$




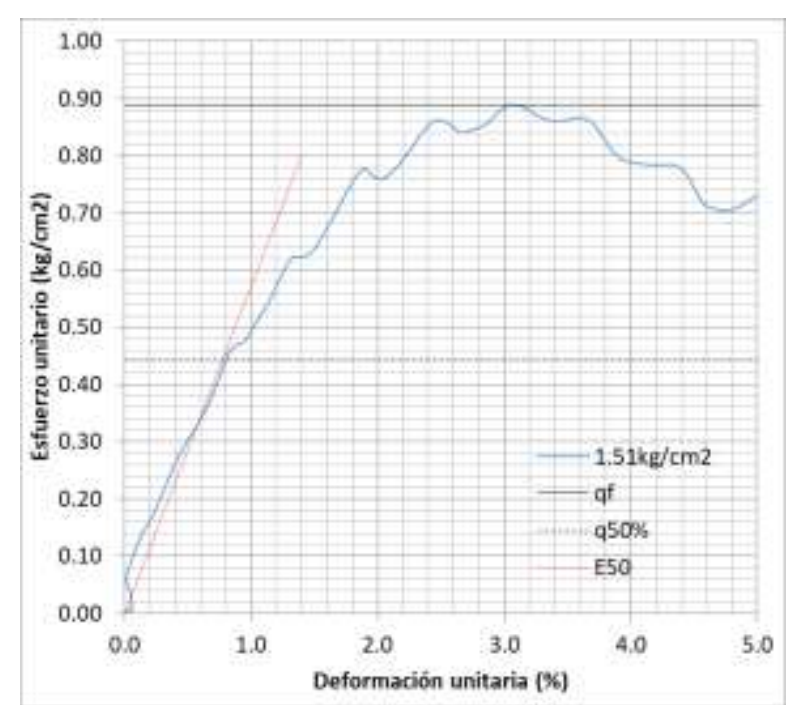

Figura 21. ${ }_{50}^{\text {ref }}$ para una presión de confinamiento

$$
\sigma_{3}=0.75 \mathrm{~kg} / \mathrm{cm}^{2}
$$

Para definir $E_{50}^{\text {ref }}$ se emplea el ensayo edométrico, dando como resultado:

Tabla 12. Cálculo de $E_{\text {oed }}^{r e f}$

Angulo de fricción interna $\varnothing=$ 7

Presión referencia $\mathrm{kg} / \mathrm{cm}^{2}=$

OCR

m

E5o ref $\mathrm{kg} / \mathrm{cm}^{2}=$

Kocn

Eoed ref $\left(\mathrm{kg} / \mathrm{cm}^{2}\right)=$
El parámetro $E_{u r}^{r e f}$ se calculó de acuerdo con la ecuación 19, con un resultado:

Tabla 13. Cálculo de $E_{u r}^{\text {ref }}$

\begin{tabular}{lc}
\hline \multicolumn{2}{c}{ Eur ref } \\
\hline Índice hinchamiento Cs & 0.01 \\
Relación de vacíos e0 & 0.59 \\
Konc & 1 \\
$\mathrm{E} 50 \mathrm{~kg} / \mathrm{cm}^{2}=$ & 47.95 \\
$\mathrm{G} \mathrm{kg} / \mathrm{cm}^{2}=$ & 17.98 \\
Poisson $\mu=$ & 0.33 \\
Presión referencia $\mathrm{kg} / \mathrm{cm}^{2}=$ & 1.02 \\
Eurref $(\mathbf{k g} / \mathrm{cm} 2)=$ & $\underline{\mathbf{1 6 5 . 9}}$ \\
\hline
\end{tabular}

La tabla 10 sirve para un estrato que va desde 0.00 a $-3.50 \mathrm{~m}$ y la tabla 11 es para el estrato de $-3.50 \mathrm{~m}$ hasta $-10.0 \mathrm{~m}$.

Tabla 14. Parámetros para el modelo HSSMALL del primer estrato

\begin{tabular}{|c|c|c|}
\hline Parámetro & Descripción & Valores \\
\hline$\mu$ & Coeficiente de Poisson & 0.33 \\
\hline yhum & Peso unitario húmedo & $17.65 \mathrm{KN} / \mathrm{m}^{3}$ \\
\hline c & Cohesión & $20.26 \mathrm{KN} / \mathrm{m}^{3}$ \\
\hline$\varnothing$ & Ángulo de fricción & 7.03 \\
\hline Ysat & Peso unitario saturado & $19.613 \mathrm{KN} / \mathrm{m}^{3}$ \\
\hline eo & Relación de vacíos inicial & 0.59 \\
\hline $\mathrm{k}$ & $\begin{array}{l}\text { Coeficiente de permeabi- } \\
\text { lidad }\end{array}$ & $1.18 \mathrm{E}-08 \mathrm{~m} / \mathrm{seg}$ \\
\hline E50 ref & $\begin{array}{l}\text { Módulo de elasticidad se- } \\
\text { cante en ensayos triaxiales }\end{array}$ & $4702.66 \mathrm{KN} / \mathrm{m}^{2}$ \\
\hline Eoed ref & $\begin{array}{l}\text { Módulo de elasticidad } \\
\text { secante en ensayos edo- } \\
\text { métricos }\end{array}$ & $4824.69 \mathrm{KN} / \mathrm{m}^{2}$ \\
\hline Eref ur & $\begin{array}{l}\text { Módulo de elasticidad de } \\
\text { carga y descarga en ensa- } \\
\text { yos cíclicos }\end{array}$ & $16267.12 \mathrm{KN} / \mathrm{m}^{2}$ \\
\hline Rf & Relación de falla & 0.90 \\
\hline oref & Esfuerzo de referencia & $100.03 \mathrm{KN} / \mathrm{m}^{2}$ \\
\hline
\end{tabular}




\begin{tabular}{|c|c|c|c|c|c|}
\hline Parámetro & Descripción & Valores & Parámetro & Descripción & Valores \\
\hline \multirow[t]{2}{*}{$\mathrm{m}$} & \multirow{2}{*}{$\begin{array}{l}\text { Dependencia de la rigidez } \\
\text { al estado de esfuerzos }\end{array}$} & \multirow[t]{2}{*}{0.66} & \multirow{3}{*}{ OCR } & Ángulo de dilatancia & 2.04 \\
\hline & & & & \multirow{2}{*}{$\begin{array}{l}\text { Relación de sobre conso- } \\
\text { lidación }\end{array}$} & \multirow{2}{*}{3.99} \\
\hline konc & $\begin{array}{l}\text { Coeficiente de suelo en } \\
\text { reposo }\end{array}$ & 1.039579 & & & \\
\hline$\Psi$ & Ángulo de dilatancia & 2.34 & Vs & $\begin{array}{l}\text { Velocidades de ondas de } \\
\text { corte }\end{array}$ & 248.00 \\
\hline OCR & $\begin{array}{l}\text { Relación de sobre conso- } \\
\text { lidación }\end{array}$ & 3.99 & Go & $\begin{array}{l}\text { Módulo cortante máxi- } \\
\text { mo }\end{array}$ & $\begin{array}{c}110047.27 \mathrm{KN} / \\
\mathrm{m}^{2}\end{array}$ \\
\hline Vs & Velocidades de ondas de & 248.00 & IP & Índice plástico & 15.00 \\
\hline Go & Módulo cortante máximo & $110662.06 \mathrm{KN} / \mathrm{m}^{2}$ & yO.7 & & 0.0001788566 \\
\hline IP & Índice plástico & 15.00 & & & \\
\hline yO.7 & & 0.0001788566 & & & \\
\hline
\end{tabular}

\subsection{Acelerograma escalado}

Tabla 15.Parámetros para el modelo HSSMALL del segundo estrato

\begin{tabular}{|c|c|c|}
\hline Parámetro & Descripción & Valores \\
\hline$\mu$ & Coeficiente de Poisson & 0.33 \\
\hline yhum & Peso unitario húmedo & $17.55 \mathrm{KN} / \mathrm{m}^{3}$ \\
\hline $\mathrm{c}$ & Cohesión & $17.36 \mathrm{KN} / \mathrm{m}^{2}$ \\
\hline$\varnothing$ & Ángulo de fricción & 6.11 \\
\hline Ysat & Peso unitario saturado & $19.221 \mathrm{KN} / \mathrm{m}^{3}$ \\
\hline eo & Relación de vacios inicial & 0.59 \\
\hline $\mathrm{k}$ & $\begin{array}{l}\text { Coeficiente de permea- } \\
\text { bilidad }\end{array}$ & $1.18 \mathrm{E}-08 \mathrm{~m} / \mathrm{seg}$ \\
\hline E50 ref & $\begin{array}{l}\text { Módulo de elasticidad } \\
\text { secante en ensayos } \\
\text { triaxiales }\end{array}$ & $4788.76 \mathrm{KN} / \mathrm{m}^{2}$ \\
\hline Eoed ref & $\begin{array}{l}\text { Módulo de elasticidad } \\
\text { secante en ensayos edo- } \\
\text { métricos }\end{array}$ & $4899.78 \mathrm{KN} / \mathrm{m}^{2}$ \\
\hline Eref ur & $\begin{array}{l}\text { Módulo de elasticidad de } \\
\text { carga y descarga en ensa- } \\
\text { yos cíclicos }\end{array}$ & $16333.78 \mathrm{KN} / \mathrm{m}^{2}$ \\
\hline $\mathrm{Rf}$ & Relación de falla & 0.90 \\
\hline oref & Esfuerzo de referencia & $100.03 \mathrm{KN} / \mathrm{m}^{2}$ \\
\hline $\mathrm{m}$ & $\begin{array}{l}\text { Dependencia de la rigi- } \\
\text { dez al estado de esfuerzos }\end{array}$ & 0.66 \\
\hline konc & $\begin{array}{l}\text { Coeficiente de suelo en } \\
\text { reposo }\end{array}$ & 1.0 \\
\hline
\end{tabular}

Se ha selezcionado el sismo de Zonocoto registrado en laestación EPN y se haocupado las dos componentes sísmicas en la direc ción E y N [24]. Este sismo ocurrió el 17 de febrer ) del 2017 [25]. A los acelerogramas se aplicóla co rección de línea base de ti so polinómica cúbica y cuencias tipo Butterworth $(0.10 \mathrm{~Hz}$ y $25 \mathrm{~Hz})$ [27].

$\left(y=a 0+a 1 x+a 2 x^{2}+a 3 x^{3}\right)$

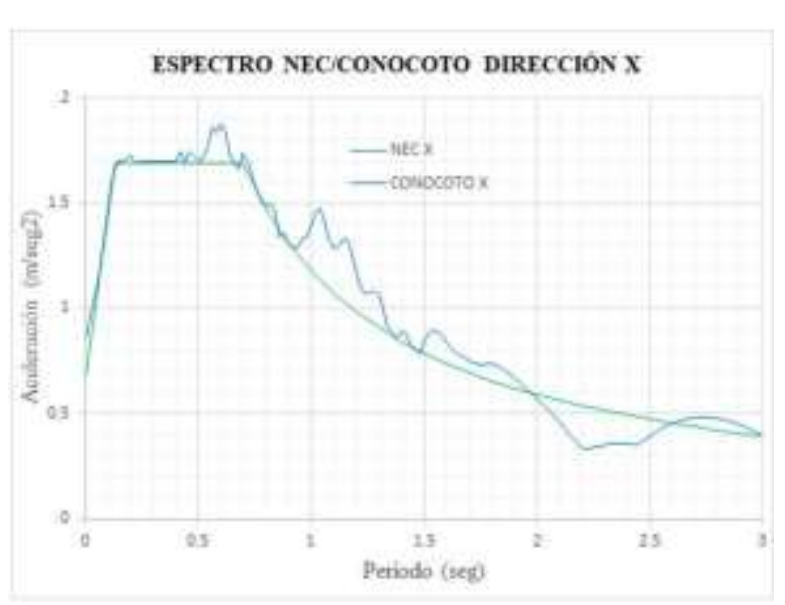




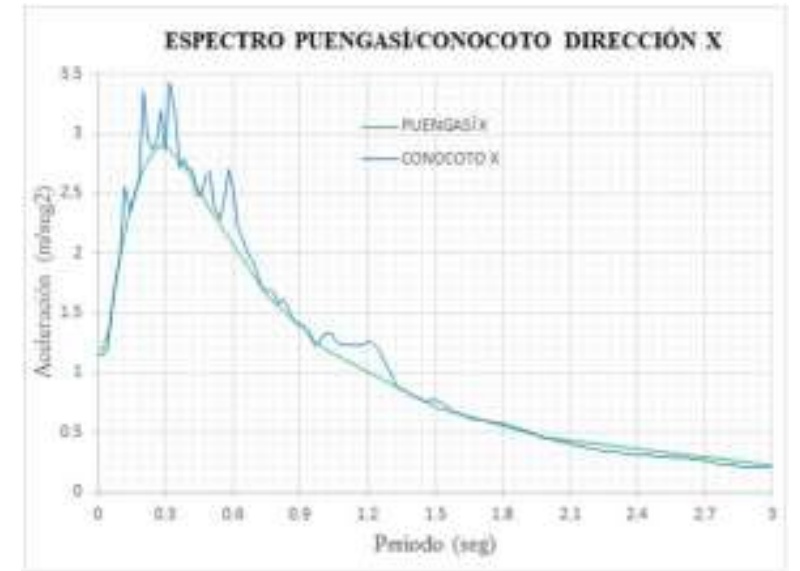

Figura 22. Acelerograma transformados a espectros escalados, en dirección X.

Como se aprecia en la figura, se observa el acelerograma de Conocoto escalado para los diferentes espectros. A continuación, el siguiente paso es transformar el espectro en un acelerograma.
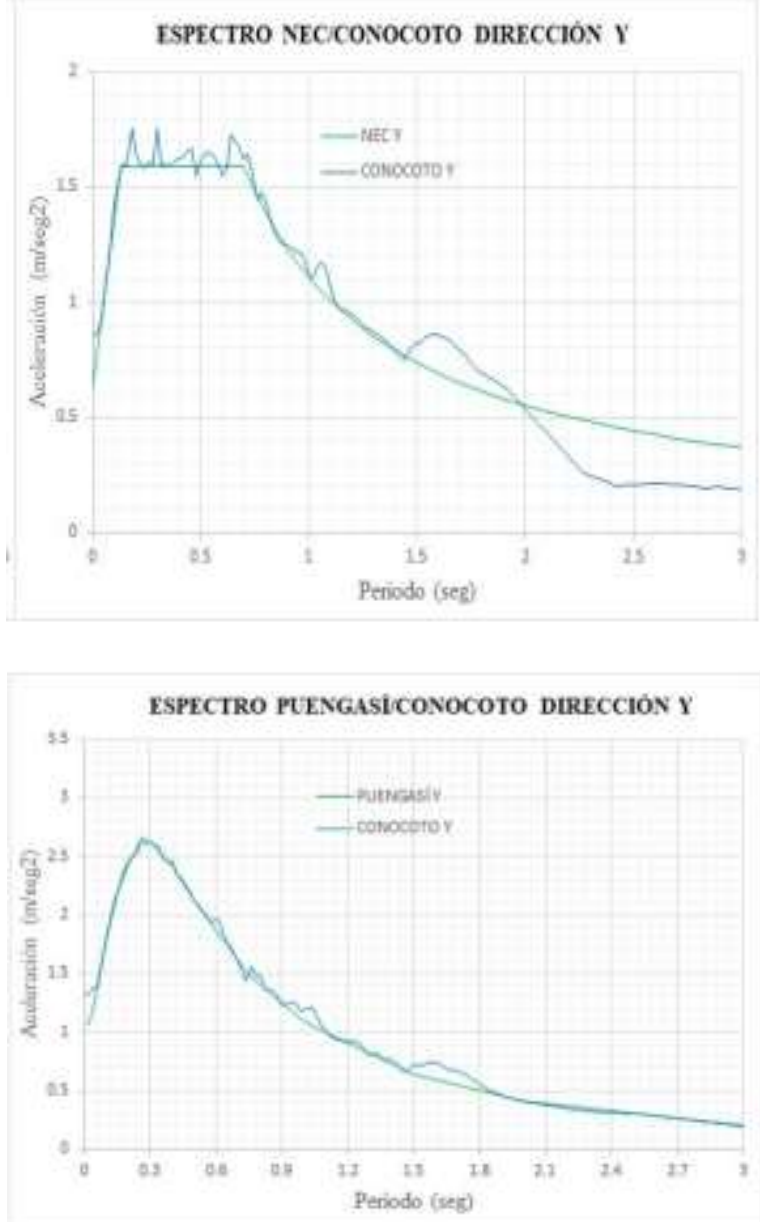

Figura 23. Acelerograma transformados a espectros y escalados, en dirección Y.

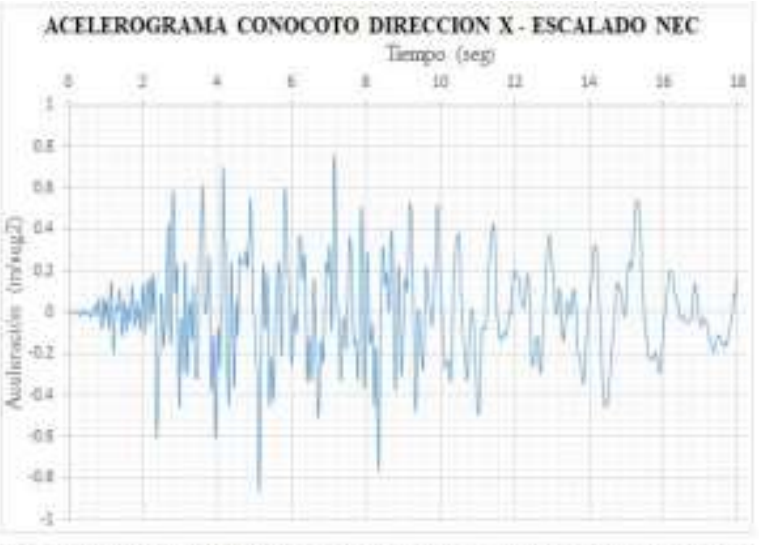

ACELEROGRAMA CONOCOTO DIRECCION X-ESCALADO PUENGASI

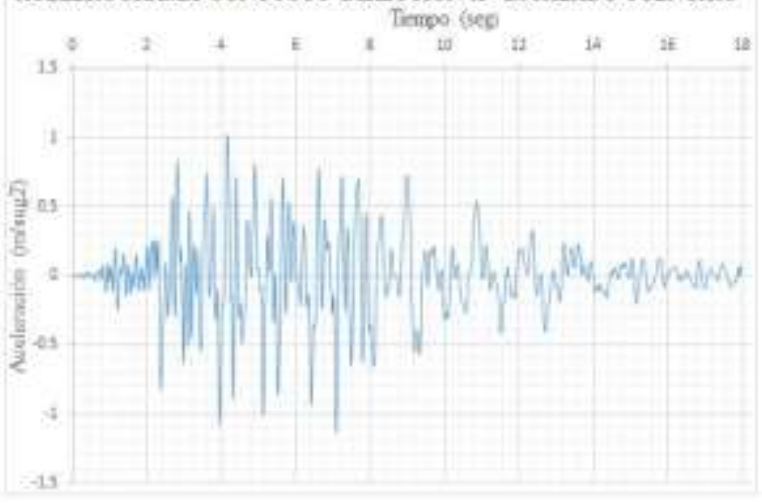

Figura 24. Acelerogramas escalados de acuerdo con los espectros y Puengasí, dirección X [24].
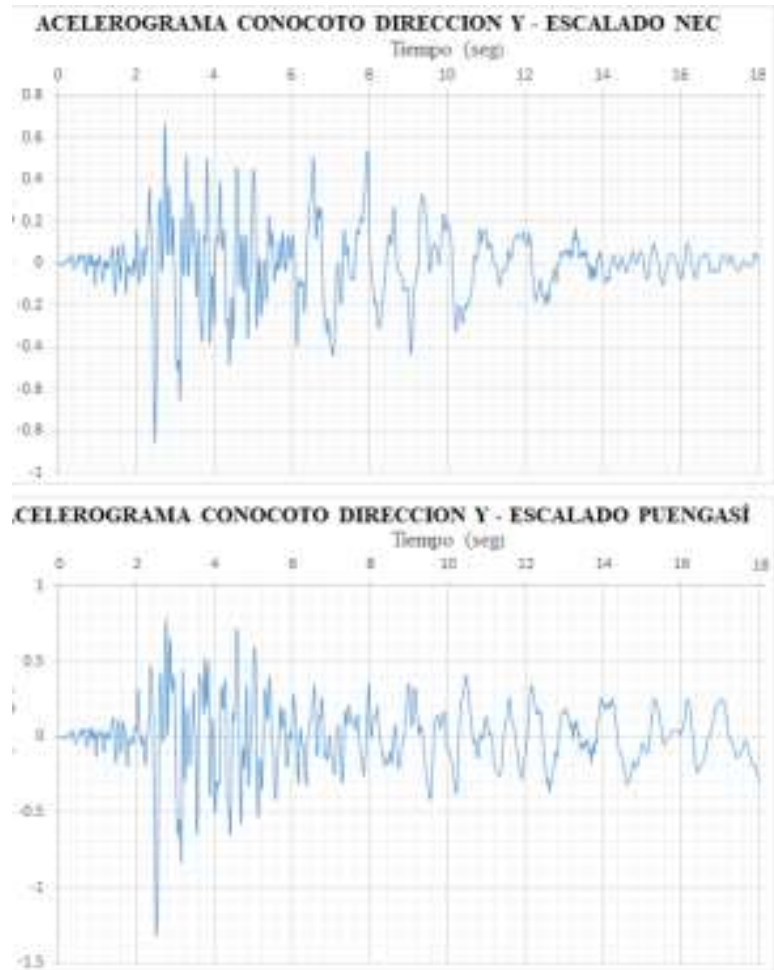

Figura 25. Acelerogramas escalados de acuerdo con los espectros [22] y Puengasí, dirección Y. 


\subsection{Modelo con elementos finitos}

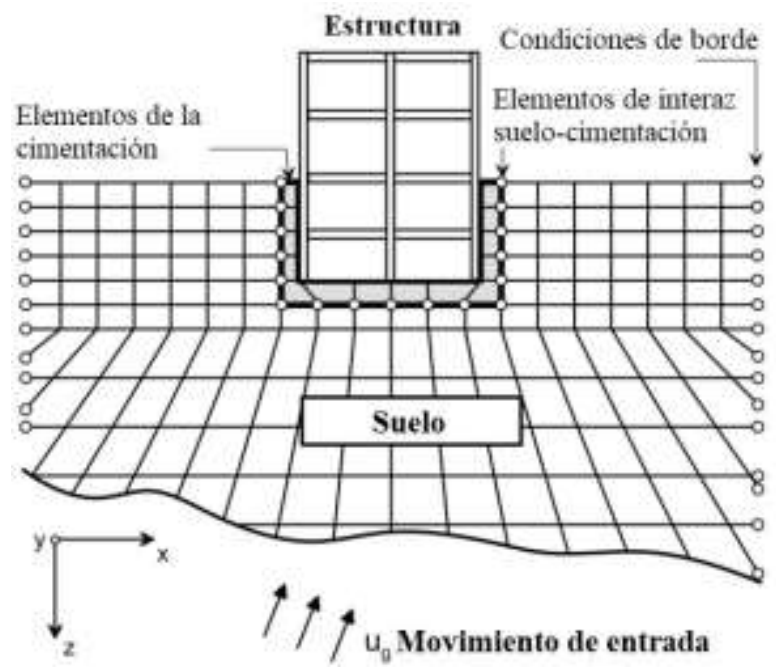

Figura 26. Modelo con elementos finitos

Después de haber definido todos los parámetros para la aplicación de un modelo elasto-plástico del suelo, se procede con el análisis en elementos finitos. De la misma manera se ingresa los espectros de respuesta.

La interfaz es la parte que realiza la interacción suelo estructura e involucra dos parámetros dentro de su cálculo la rigidez tangente y normal, cuyo cálculo se lo realiza a partir de las siguientes ecuaciones [26]:

$$
\begin{aligned}
& K n=\frac{E_{\text {oed }}}{L . t v} \\
& K t=\frac{G i}{L . t v}
\end{aligned}
$$

$$
G i=\text { R. Gsoil }
$$

Gsoil $=\frac{E}{2 \cdot\left(2+v_{i}\right)}$

Donde:

Eoed : Es el módulo de elasticidad de ensayos edométricos

$v_{i} \quad$ : Coeficiente de Poisson de la interfaz, cuyo valor recomendado es de 0.45
$L \quad$ : es la longitud de análisis, que por default se le asigna $1 \mathrm{~m}$

tv : Es el espesor virtual de la interfaz (Generalmente tiene un valor entre $0.01 \sim 0.1$, cuanto mayor sea la diferencia de rigidez entre el suelo y la estructura, menor será el valor)

$R$ : Factor de reducción estructural cuyos valores son:

$\begin{array}{ll}\text { Arena/Acero } & =0.6-0.7 \\ \text { Arcilla/Acero } & =0.5 \\ \text { Arena/Hormigón } & =0.80-1.00 \\ \text { Arcilla/Hormigón } & =0.70-1.00\end{array}$

Por lo tanto, se ha definido los siguientes valores de $R=0.7$, Eoed $=80.372 \mathrm{~kg} / \mathrm{cm} 2$ y tv $=0.03$, dando los siguientes valores de $\mathrm{Kn}$ y Kt:

$\mathrm{Kn}=2679.07 \mathrm{~kg} / \mathrm{cm}^{3}=262726.81 \mathrm{kN} / \mathrm{m}^{3}$

$K t=646.671 \mathrm{~kg} / \mathrm{cm}^{3}=63416.81 \mathrm{kN} / \mathrm{m}^{3}$

Los modos de vibración de este modelo se identifi-

\begin{tabular}{|c|c|}
\hline Modo & Periodo (seg) \\
\hline 1 & 1.54 \\
\hline 2 & $\underline{1.53}$ \\
\hline 3 & 1.50 \\
\hline 4 & 1.47 \\
\hline 5 & $\underline{1.43}$ \\
\hline 6 & $\underline{1.42}$ \\
\hline 7 & 1.39 \\
\hline 8 & 1.38 \\
\hline 9 & $\underline{1.36}$ \\
\hline 10 & $\underline{1.32}$ \\
\hline 11 & $\underline{1.29}$ \\
\hline 12 & $\underline{1.22}$ \\
\hline 13 & 1.15 \\
\hline 14 & $\underline{1.13}$ \\
\hline
\end{tabular}
can en la siguiente tabla.

Tabla 16. Periodos de vibración del Modelo C 


\begin{tabular}{lll}
\hline \begin{tabular}{ll}
15 \\
\cline { 1 - 1 }
\end{tabular} & & $\underline{1.13}$ \\
\hline 16 & & 1.04 \\
\hline 18 & 1.02 \\
\hline 19 & & 1.00 \\
\hline 20 & & 0.98 \\
\hline 21 & $\underline{0.96}$ \\
\hline 22 & $\underline{0.94}$ \\
\hline 23 & $\underline{0.93}$ \\
\hline 24 & $\underline{0.90}$ \\
\hline
\end{tabular}

Las derivas máximas se indican a continuación:

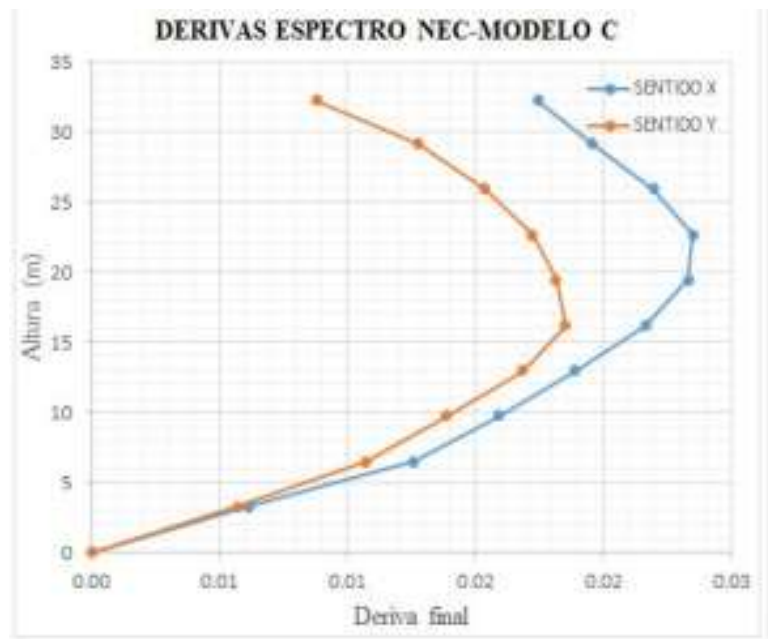

Figura 27. Derivas máximas del Modelo C, con Espectro NEC



Figura 28. Derivas máximas del Modelo C, con Espectro Puengasí

\section{Para el espectro NEC:}

$\Delta \mathrm{x}=0.024$

$\Delta \mathrm{y}=0.019$

\section{Para el espectro Puengasí:}

$\Delta \mathrm{x}=0.021$

$\Delta \mathrm{y}=0.018$

Valores superiores a 0.02 que reporta la [21].

\section{Comparación entre los tres métodos}

El periodo de vibración es un indicativo de la rigidez de la estructura, pero como se ha visto hasta ahora también está involucrado el suelo bajo la cimentación. Solo se presentan los tres primeros modos de vibración, ya que como se analizó en el método de la subestructura más del $90 \%$ de la participación modal se encuentran en estas tres primeras formas modales.

Tabla 17. Resumen de los periodos de vibración de la estructura para los tres métodos

\begin{tabular}{cccc}
\hline \multirow{2}{*}{ Modo } & \multicolumn{3}{c}{ Periodo(seg) } \\
\cline { 2 - 4 } & Modelo A & Modelo B & Modelo C \\
\hline 1 & 1.06 & 1.55 & 1.54 \\
\hline 2 & 0.98 & 1.43 & 1.53 \\
\hline 3 & 0.89 & 1.24 & 1.50 \\
\hline
\end{tabular}

Comparando el primer modo con el modelo A, existe un $46 \%$ con el Modelo B y $45 \%$ el Modelo C. En otras palabras, realizando un análisis con base rígida (Modelo A) en un suelo blando como el realizado en esta investigación, se comete un gran error que a posterior dará resultados positivos falsos (derivas dentro parámetros permitidos, secciones y armados de acero de elementos estructurales, fenómeno de resonancia, etc.).

El periodo de vibración natural del depósito es 1.022 segundos y para estimar si la estructura se encuentra en resonancia se debe evaluar hasta el $90 \%$ de la participación modal y en este caso invo- 
lucra los tres primeros modos, estableciendo que solo el modelo A presenta este fenómeno.

Las derivas máximas de piso se indican a continuación:

Tabla 18. Resumen de derivas máximas de la estructura para los tres métodos

\begin{tabular}{clll}
\hline \multicolumn{4}{c}{ Derivas máximas X/Y } \\
\hline \multirow{2}{*}{ TIPO } & Modelo A & Modelo B & Modelo C \\
\hline \multirow{2}{*}{ NEC } & $0.01136 /$ & $0.02 /$ & $0.024 /$ \\
& 0.00981 & 0.0176 & 0.019 \\
\hline \multirow{2}{*}{ Puengasí } & $0.01102 /$ & $0.0188 /$ & $0.021 /$ \\
& 0.009 & 0.0168 & 0.018 \\
\hline
\end{tabular}

Según la [21, p. 40] este valor no debe exceder a 0.02 , por lo tanto con el modelo $\mathrm{C}$ no cumple satisfactoriamente las derivas de piso para el caso del espectro de la NEC y Puengasí.

\section{Conclusiones}

- Para estimar el espectro de respuesta de sitio mediante la plataforma virtual del PEER [19], es necesario contar con una caracterización geológica de las fuentes sísmicas (fallas).

- El empotramiento perfecto es una manera de realizar la interacción suelo estructura, pero esto significa que se asume que el suelo es rígido.

- Los resultados más fiables de realizar una interacción suelo estructura es con el uso de las funciones de impedancia (Modelo B) y mediante el método directo (Modelo C), que estimaron valores de periodos de vibración y derivas de piso muy cercanas entresí.

- Es necesario diferenciar entre el coeficiente de balasto y funciones de impedancia, la única similitud entre ambas es el uso de resortes (rigidez) para interpretar al suelo., después de eso no existe ninguna similitud debido a que la primera se obtiene mediante un ensayo estático, mientras que la segunda incluye el uso de parámetros dinámicos del suelo como el módulo cortante máximo (Gmax) y modulo cortante secante (Gsec) y cuya estimación más cercana se realiza a partir de las velocidades de ondas de corte (Vs).
- Cuando se emplee el Vs se debe tomar en cuenta la profundidad en donde se colocará la cimentación y no confundir con el Vs30 que es la velocidad promedio a $30 \mathrm{~m}$ de profundidad. En un ensayo de velocidades de ondas de corte se grafica la profundidad versus Vs30, de esta manera se evita el error.

- El espectro de la NEC-SE-DS, es satisfactorio para esta investigación, ya que al comparar con los resultados obtenidos con el espectro de Puengasí, existe similitud de valores (Ver Tabla 5.16).

- Se ha demostrado en esta investigación, que al no considerar la ISE (modelo A), se genera errores muy graves en el diseñoestructural.

\section{Referencias}

[1] G. M. Martin, «Proceedings of the NCEER/SEAOC/BSSC Workshop on Site Response during Earthquakes and Seismic Code Revisions,» Univ. of Southern Calif, 1994.

[2] Pacific Earthquake Engineering Research Center, «PER Ground Motion Database,» 2509 2019. [En línea]. Available: www. ngawest2.berkeley.edu.

[3] R. F. Obrzud y A. Truty, THE HARDENING SOIL MODEL-A PRACTICAL GUIDEBOOK, Préverenges: Structures, 2018.

[4] T. Schanz, P. Vermeer y P. Bonnier, «The hardening soil model: Formulation and verification,» Beyond 2000 in Computational Geotechnics , p. 16, 1998.

[5] PLAXIS, Material Models Manual, 2017.

[6] A. Alvarado, Neotectonique et cinematique de la deformation continentae en Equador, Arrete: Universite de Grenoble, 2012.

[7] J. Biarez y P. Hicher, «Elementary Mechanics of Soil Behaviour. Saturated remoulded soils,» 1994.

[8] ASCE 7, Seismic Evaluation and Retrofit of Existing Buildings, Reston, Virginia: American Society of Civil Engineers, 2016. 
[9] Y.Nakamura, «A METHOD FOR ESTIMATING THE DYNAMIC CHARACTERIZATION OF THE SUBSOIL USING MICROTREMORS ON THE SOIL SURFACE,» p. 33, 1989.

[10] Pacific Earthquake Engineering Research Center, «PER Ground Motion Database,» 0406 2019. [En línea]. Available: www. ngawest2.berkeley.edu.

[11] R. Aguiar, Espectros de control para la ciudad de Quito-Ecuador (Obtención de espectros mediante modelos determinísticos asociados a las fallas ciegas inversas de Quito), Barcelona: A.H. Barbat, 2015.

[12] H. Parra , Desarrollos Metodológicos y aplicaciones hacia el calculo de la peligrosidad sísmica en el Ecuador continental y estudio e riesgo sísmico en la ciudad de Quito, Madrid: Desarrollos Metodológicos y aplicaciones hacia el calculo de la peligrosidad sísmica en el Ecuador continental y estudio e riesgo sísmico en la ciudad de Quito, 2016.

[13] K. Campbell y Y. Borzognia, NGA-Wets2 Campbell-Bozornia Ground Motion Model for the Horizontal Components of PGA, PGV and 5\% Damped Elastic

Pseudo-Acceleration Response Spectra for Periods Ranging from 0.01 to $10 \mathrm{sec}$, Berkeley: Pacific Earthquake Engineering Research Center, 2013.

[14] J. Kaklamanos, L. G. Baise y D. M. Boore, «Estimating Unknown Input Parameters when Implementing the NGA Ground-Motion Prediction Equations in Engineering Practice,» Earthquake Engineering Research Institute, p. 17, 2011.

[15] N. Abrahamson, W. Silva y R. Kamai, «Summary of the ASK14 Ground-Motion Relation for Active Crustal Regions,» Earthquake Spectra, p. 39, 2014.
[16] N. Abrahamson, W. Silvia y K. R.,Update of the AS08 Ground-Motion Prediction Equation Based on the NGA-West2 Data Set, Berkeley: Pacific Earthquake Engineering Research Center, 2013.

[17] B. Chiou y R. Youngs, Update of the Chiou and Youns NGA Ground Motion Model for Average Horizontal Component of Peak Ground Motion and Response Spectra, Berkeley: Pacific Earthquake Engineering Research Center, 2013.

[18] PEER, Users Manual for the PEER Ground Motion Database Web Application, Pacific Earthquake Engineering Research Center, 2011.

[19] NEC-SE-DS, Norma Ecuatoriana de la Construcción-Capitulo de Peligro Sísmico y Diseño Simoresistente, Quito, 2015.

[20] NEC-SE-DS, Norma Ecuatoriana de la Construcción-Capitulo de Peligro Sísmico y Diseño Simoresistente, Quito, 2015.

[21] NIST GCR 12-917-21, Solil-Structure Interaction for Building Structures, Gaithersburg: U.S Department of Commerce, 2012.

[22] A. Chopra, Dinámica de estructuras, México Df: Pearson, 2014.

[23] NEC-SE-DS, Norma Ecuatoriana de la Construcción-Capitulo de Peligro Sísmico y Diseño Simoresistente, Quito, 2015.

[24] «Instituto Geofísico,» 0210 2019. [En línea]. Available: www.igepn.edu.ec.

[25] MIDAS, New experience Geotechnical analysis System, 2019.

[26] U.S Geological Survey \& Esc. Politécnica Nacional, «Mapa de Fallas y Pliegues Cuarternarias de Ecuador y Regiones Oceanicas Adyacentes,» USGS, Denver \& Quito, 2003. 